\title{
Linear stability of blowup solution of incompressible Keller-Segel-Navier-Stokes system
}

Yan Yan ${ }^{1 *}$ and Hengyan Li2*

"Correspondence:
yanyan_1984@163.com;
lihengyan@ncwu.edu.cn
'School of Mathematics and
Information Science, Henan
University of Economics and Law,
Zhengzhou, P.R. China
2School of Mathematics and
Information Sciences, North China
University of Water Resources and
Electric Power, Zhengzhou 450011,
P.R. China

\begin{abstract}
In this paper, we consider the linear stability of blowup solution for incompressible Keller-Segel-Navier-Stokes system in whole space $\mathbb{R}^{3}$. More precisely, we show that, if the initial data of the three dimensional Keller-Segel-Navier-Stokes system is close to the smooth initial function $\left(0,0, \mathbf{u}_{s}(0, x)\right)^{T}$, then there exists a blowup solution of the three dimensional linear Keller-Segel-Navier-Stokes system satisfying the decomposition

$$
(n(t, x), c(t, x), \mathbf{u}(t, x))^{T}=\left(0,0, \mathbf{u}_{s}(t, x)\right)^{T}+\mathcal{O}(\varepsilon), \quad \forall(t, x) \in\left(0, T^{*}\right) \times \mathbb{R}^{3}
$$

in Sobolev space $H^{5}\left(\mathbb{R}^{3}\right)$ with $s=\frac{3}{2}-5 a$ and constant $0<a \ll 1$, where $T^{*}$ is the maximal existence time, and $\mathbf{u}_{s}(t, x)$ given in (Yan 2018) is the explicit blowup solution admitted smooth initial data for three dimensional incompressible Navier-Stokes equations.
\end{abstract}

MSC: $35 \mathrm{~K} 15 ; 74 \mathrm{H} 35 ; 35 \mathrm{~B} 35$

Keywords: Keller-Segel-Navier-Stokes system; Blowup solution; Stability

\section{Introduction and main results}

The Keller-Segel system coupled to the incompressible Navier-Stokes equations arises from a biological process in which cells move towards a chemically more favorable environment [20]. In this paper, we consider the blowup analysis for the three dimensional Keller-Segel-Navier-Stokes system

$$
\left\{\begin{array}{l}
n_{t}+\mathbf{u} \cdot \nabla n=\Delta n-\chi \nabla \cdot(n \nabla c)+\sigma n-\mu n^{2} \\
c_{t}+\mathbf{u} \cdot \nabla c=\Delta c-c+n \\
\mathbf{u}_{t}+\mathbf{u} \cdot \nabla \mathbf{u}=\Delta \mathbf{u}-\nabla P+n \nabla \Phi \\
\nabla \cdot \mathbf{u}=0
\end{array}\right.
$$

where $\forall(t, x) \in \mathbb{R}^{+} \times \mathbb{R}^{3}, \sigma \in \mathbb{R}$ and $\mu>0$ are given parameters, the constant $\chi$ is the chemotactic sensitivity. We assume $\chi>0$ presuming that cells move toward increasing

(c) The Author(s) 2021. This article is licensed under a Creative Commons Attribution 4.0 International License, which permits use, sharing, adaptation, distribution and reproduction in any medium or format, as long as you give appropriate credit to the original author(s) and the source, provide a link to the Creative Commons licence, and indicate if changes were made. The images or other third party material in this article are included in the article's Creative Commons licence, unless indicated otherwise in a credit line to the material. If material is not included in the article's Creative Commons licence and your intended use is not permitted by statutory regulation or exceeds the permitted use, you will need to obtain permission directly from the copyright holder. To view a copy of this licence, visit http://creativecommons.org/licenses/by/4.0/. 
concentrations of the signal substance which is produced by themselves. The unknown scalar functions $n(t, x): \mathbb{R}^{+} \times \mathbb{R}^{3} \rightarrow \mathbb{R}$ and $c(t, x): \mathbb{R}^{+} \times \mathbb{R}^{3} \rightarrow \mathbb{R}$ denote the population density and the signal concentration, respectively, the vector function $\mathbf{u}(t, x): \mathbb{R}^{+} \times \mathbb{R}^{3} \rightarrow$ $\mathbb{R}^{3}$ denotes the $3 \mathrm{D}$ velocity field of the fluid, $P(t, x): \mathbb{R}^{+} \times \mathbb{R}^{3} \rightarrow \mathbb{R}$ stands for the pressure in the fluid. Moreover, the pressure $P(t, x)$ is determined by the formula

$$
P(t, x)=-\triangle^{-1}\left(\sum_{i, j=1}^{3} \frac{\partial v_{i}}{\partial x_{j}} \frac{\partial v_{j}}{\partial x_{i}}-\nabla \cdot(n \nabla \Phi)\right) .
$$

$\Phi(x)$ is a given potential function accounting for the effects of external forces such as gravity. The divergence free condition in the last equation of (1.1) guarantees the incompressibility of the fluid.

Chemotaxis, as a mechanism of the partially-oriented movement of cells in response to a chemical signal, plays an important role in various biological process [3, 11]. Tuval et al. [26] observed large-scale convection patterns in a water drop sitting on a glass surface containing oxygen-sensitive bacteria, oxygen diffusing into the drop through the fluid-air interface. They established a mathematical model, the so-called chemotaxis-fluid system, to describe the dynamics of swimming bacteria, Bacillus subtilis. It has the form

$$
\left\{\begin{array}{l}
n_{t}+\mathbf{u} \cdot \nabla n=\Delta n-\nabla \cdot(n \chi(c) \nabla c), \\
c_{t}+\mathbf{u} \cdot \nabla c=\Delta c-n \kappa(c), \\
\mathbf{u}_{t}+\kappa(\mathbf{u} \cdot \nabla \mathbf{u})=\Delta \mathbf{u}-\nabla P+n \nabla \Phi, \\
\nabla \cdot \mathbf{u}=0, \quad \forall(t, x) \in \mathbb{R}^{+} \times \mathcal{D},
\end{array}\right.
$$

where $\mathcal{D}$ is a smooth bounded domain or $\mathbb{R}^{3}$. Lorz [19] first showed the local existence of weak solutions for the two and three dimensional system (1.1) with nonflux and inhomogeneous Dirichlet boundary conditions, respectively. Chae-Kang-Lee [6] gave the local existence of the smooth solution in the cases of two and three dimensions and global existence of classical solution for the two dimensional case for system (1.1). Winkler [31] asserted that a solution of the two dimensional chemotaxis-NavierStokes system in a bounded convex domain $\Omega$ stabilizes to the spatially uniform equilibrium $\left(\frac{1}{|\Omega|} \int_{\Omega} n_{0}(x) d x, 0,0\right)^{T}$ in $\mathbb{L}^{\infty}(\Omega)$. Zhang-Zheng [40] used a microlocal analysis to obtain the global existence and uniqueness of weak solutions for a two dimensional chemotaxis-Navier-Stokes system in $\mathbb{R}^{3}$ for a large class of initial data. One can also refer to $[7,18,28,29]$ for more results on this two dimensional model. For the spatially three dimensional case, Tao-Winkler [25] constructed locally bounded global solutions in a chemotaxis-Stokes system with nonlinear diffusion. Winkler [33] established the global weak existence theory to a chemotaxis-Navier-Stokes system in a smooth bounded convex domain under a homogeneous Neumann boundary condition. With some relaxation time, Winkler [34] has shown this model admitted eventual energy solutions, meanwhile, he got such eventual energy solutions $(n, c, \mathbf{u}) \rightarrow\left(\bar{n}_{0}, 0,0\right)$ uniformly in a smooth bounded convex domain after the waiting time. Due to spatially limitation, we do not list all of the interesting papers on this kind of models.

Since the Keller-Segel-Navier-Stokes system plays an important role in bioconvection processes, it has attracted great interest also at the level of mathematical theory [2, 3]. 
Wang [27] showed the global existence of weak solutions for a three dimensional KellerSegel-Navier-Stokes system with subcritical sensitivity. Winkler [35] proved that the three dimensional model with logistic source admits global weak solutions and asymptotic stabilization in a smooth bounded convex domain with homogeneous Dirichlet boundary conditions. At present, there are extreme difficulties to study the properties of solutions for system (1.1). One of the reasons is the question of finite time singularity/global regularity for three dimensional incompressible Navier-Stokes equations, one of the most important open problems in mathematical fluid mechanics [9]. On the one hand, even if we get rid of the effect of a fluid $(\mathbf{u} \equiv 0)$, the question whether blowup may occur in the three dimensional Keller-Segel system

$$
\begin{cases}n_{t}=\Delta n-\chi \nabla \cdot(n \nabla c)+\sigma n-\mu n^{2}, & (t, x) \in \mathbb{R}^{+} \times \Omega, \\ c_{t}=\Delta c-c+n, & (t, x) \in \mathbb{R}^{+} \times \Omega,\end{cases}
$$

with small positive constant $\mu$ is still open [35]. Here $\Omega$ is a smooth bounded domain in $\mathbb{R}^{3}$. Lankeit [15] got the global existence of weak solutions for system (1.3). Winkler [30] proved that system (1.3) can lead to a finite time explosion even for the case $\sigma=\mu=0$. One can refer to $[12-14,32]$ for more results on this kind of models.

On the other hand, if we set $n(t, x)=c(t, x) \equiv 0$, then the Keller-Segel-Navier-Stokes system (1.1) is reduced to the $3 D$ incompressible Navier-Stokes equations

$$
\begin{aligned}
& \mathbf{u}_{t}+\mathbf{u} \cdot \nabla \mathbf{u}=v \triangle \mathbf{u}-\nabla P, \\
& \nabla \cdot \mathbf{u}=0 .
\end{aligned}
$$

For these equations, Leray [16] showed there is global-forward-in-time weak solution of the initial value problem. After that, non-existence of finite energy self-similar blowup solutions in $\mathbb{L}^{3}\left(\mathbb{R}^{3}\right)$ was obtained in [22]. As is well known, Eq. (1.4) admits a simplest nontrivial stationary solution with infinite energy, i.e. the Couette flow $(y, 0,0)^{T}$. BedrossianGermain-Masmoudi [1] proved the nonlinear stability of this flow. One can refer to $[4,5,8,10,17,21,24,39]$ for more related results. Yan [36, 37] found that the three dimensional Navier-Stokes equation (1.4) admits a family of stable explicit blowup solutions with infinite energy,

$$
\begin{aligned}
\mathbf{u}_{s}(t, x) & =\left(\frac{a x_{1}}{T^{*}-t}+k x_{2}\left(T^{*}-t\right)^{2 a}, \frac{a x_{2}}{T^{*}-t}-k x_{1}\left(T^{*}-t\right)^{2 a},-\frac{2 a x_{3}}{T^{*}-t}\right)^{T}, \\
(t, x) & \in\left[0, T^{*}\right) \times \mathbb{R}^{3},
\end{aligned}
$$

with the smooth initial data

$$
\mathbf{u}_{s}(0, x)=\left(\frac{a x_{1}}{T^{*}}+k x_{2}\left(T^{*}\right)^{2 a}, \frac{a x_{2}}{T^{*}}-k x_{1}\left(T^{*}\right)^{2 a},-\frac{2 a x_{3}}{T^{*}}\right)^{T},
$$

where we have the constants $a, k \in \mathbb{R} /\{0\}$, and the positive constant $T^{*}$ is the maximal existence time. Moreover, the pressure $P$ is determined by the formula

$$
P(t, x)=-\triangle^{-1} \sum_{i, j=1}^{3} \frac{\partial u_{i}}{\partial x_{j}} \frac{\partial u_{j}}{\partial x_{i}}
$$


We supplement the $3 \mathrm{D}$ incompressible Keller-Segel-Navier-Stokes equations (1.1) with the initial data

$$
\begin{cases}n(0, x)=n_{0}(x), & x \in \mathbb{R}^{3}, \\ c(0, x)=c_{0}(x), & x \in \mathbb{R}^{3}, \\ \mathbf{u}(0, x)=\mathbf{u}_{0}(x), & x \in \mathbb{R}^{3} .\end{cases}
$$

We now state the main result.

Theorem 1.1 Let $0<a \ll \frac{1}{8}$ and $0<s<\frac{3}{2}-5 a$ be constants. Assume that $\|\Phi\|_{\mathbb{H}^{s+3}\left(\mathbb{R}^{3}\right)} \lesssim$ $R \ll 1$. The three dimensional Keller-Segel-Navier-Stokes system (1.1) admits a family of linearly stable blowup solutions

$$
(n(t, x), c(t, x), \boldsymbol{u}(t, x))^{T}=\left(0,0, \boldsymbol{u}_{s}(t, x)\right)^{T} .
$$

Throughout this paper, we denote the usual norm of $\mathbb{L}^{2}\left(\mathbb{R}^{3}\right)$ and Sobolev space $\mathbb{H}^{s}\left(\mathbb{R}^{3}\right)$ by $\|\cdot\|_{\mathbb{L}^{2}}$ and $\|\cdot\|_{\mathbb{H}^{s}}$ for $s \in \mathbb{R}^{+}$, respectively. In fact, the Sobolev space can be equivalent to be defined via Fourier transformation, thus the fractional case is contained. The norm of the Sobolev space $H^{s}\left(\mathbb{R}^{3}\right):=\left(\mathbb{H}^{s}\left(\mathbb{R}^{3}\right)\right)^{3}$ is denoted by $\|\cdot\|_{H^{s}}$. The space $\mathbb{L}^{2}\left(\left[0, T^{*}\right) ; H^{s}\left(\mathbb{R}^{3}\right)\right)$ is equipped with the norm

$$
\|u\|_{\mathbb{L}^{2}\left(\left(0, T^{*}\right) ; H^{s}\left(\mathbb{R}^{3}\right)\right)}^{2}:=\int_{0}^{T^{*}}\|u(t, \cdot)\|_{H^{s}}^{2} d t .
$$

We also introduce the function spaces

$$
\begin{aligned}
& \mathcal{C}_{0}^{s}:=\bigcap_{i=0}^{1} \mathbb{C}^{i}\left(\left(0, T^{*}\right) ; \mathbb{H}^{s-i}\left(\mathbb{R}^{3}\right)\right), \\
& \overline{\mathcal{C}}_{0}^{s}:=\bigcap_{i=0}^{1} \mathbb{C}^{i}\left(\left(0, T^{*}\right) ; H^{s-i}\left(\mathbb{R}^{3}\right)\right),
\end{aligned}
$$

with the norm

$$
\begin{aligned}
& \|u\|_{\mathcal{C}_{1}^{s}}^{2}:=\sup _{t \in\left(0, T^{*}\right)} \sum_{i=0}^{1}\left\|\partial_{t}^{i} u\right\|_{\mathbb{H}^{s-i}}^{2}, \\
& \|u\|_{\mathcal{C}_{1}^{s}}^{2}:=\sup _{t \in\left(0, T^{*}\right)} \sum_{i=0}^{1}\left\|\partial_{t}^{i} u\right\|_{H^{s-i}}^{2},
\end{aligned}
$$

respectively. The symbol $a \lesssim b$ means that there exists a positive constant $C$ such that $a \leq C b .(a, b, c)^{T}$ denotes the column vector in $\mathbb{R}^{3}$. The letter $C$ with subscripts to denote dependencies stands for a positive constant that might change its value at each occurrence. 


\section{Proof of Theorem 1.1}

The linear stability of a blowup solution is equivalent to the well-posedness of linearized equations in $\mathbb{R}^{3}$. For any $(t, x) \in\left(0, T^{*}\right) \times \mathbb{R}^{3}$, we recall the perturbation equations

$$
\left\{\begin{array}{l}
n_{t}+(\mathbf{v}+\overline{\mathbf{u}}) \cdot \nabla n=\Delta n-\chi \nabla \cdot(n \nabla c)+\sigma n-\mu n^{2} \\
c_{t}+(\mathbf{v}+\overline{\mathbf{u}}) \cdot \nabla c=\Delta c-c+n, \\
\mathbf{v}_{t}+\mathbf{v} \cdot \nabla \overline{\mathbf{u}}+\overline{\mathbf{u}} \cdot \nabla \mathbf{v}+\mathbf{v} \cdot \nabla \mathbf{v}=\nabla \bar{P}+v \Delta \mathbf{v}+n \nabla \Phi, \\
\nabla \cdot \mathbf{v}=0
\end{array}\right.
$$

with initial data

$$
\begin{cases}n(0, x)=n_{0}(x), & x \in \mathbb{R}^{3}, \\ c(0, x)=c_{0}(x), & x \in \mathbb{R}^{3}, \\ \mathbf{v}(0, x)=\mathbf{v}_{0}(x), & x \in \mathbb{R}^{3},\end{cases}
$$

and the boundary condition

$$
\left\{\begin{array}{l}
\lim _{|x| \rightarrow+\infty} n(t, x)=0 \\
\lim _{|x| \rightarrow+\infty} c(t, x)=0 \\
\lim _{|x| \rightarrow+\infty} \mathbf{v}(t, x)=0
\end{array}\right.
$$

where $(t, x) \in\left(0, T^{*}\right) \times \mathbb{R}^{3}$, the pressure $\bar{P}$ satisfies

$$
\begin{aligned}
\bar{P}(t, x)= & -\triangle^{-1}\left(\sum_{k=1}^{3}\left(\frac{\partial v_{k}}{\partial x_{k}}\right)^{2}+2 k\left(T^{*}-t\right)^{2 a}\left(\frac{\partial v_{2}}{\partial x_{1}}-\frac{\partial v_{1}}{\partial x_{2}}\right)+2 \frac{\partial v_{1}}{\partial x_{2}} \frac{\partial v_{2}}{\partial x_{1}}\right. \\
& \left.+2 \frac{\partial v_{1}}{\partial x_{3}} \frac{\partial v_{3}}{\partial x_{1}}+2 \frac{\partial v_{2}}{\partial x_{3}} \frac{\partial v_{3}}{\partial x_{2}}-\nabla \cdot(n \nabla \Phi)\right),
\end{aligned}
$$

and we have

$$
\nabla \overline{\mathbf{u}}=\left(\begin{array}{ccc}
\frac{a}{T^{*}-t} & -k\left(T^{*}-t\right)^{2 a} & 0 \\
k\left(T^{*}-t\right)^{2 a} & \frac{a}{T^{*}-t} & 0 \\
0 & 0 & -\frac{2 a}{T^{*}-t}
\end{array}\right) .
$$

Let $R \in(0,1)$ be a fixed constant. We define

$$
\mathcal{B}_{R}:=\left\{(n(t, x), c(t, x), \mathbf{v}(t, x))^{T}:\|n\|_{\mathcal{C}_{1}^{s+\frac{11}{2}}}+\|c\|_{\mathcal{C}_{1}^{s+\frac{11}{2}}}+\|\mathbf{v}\|_{\mathcal{C}_{1}^{s+\frac{11}{2}}} \leq R<1\right\}
$$

with a constant $s>0$.

Assume that fixed functions $(n(t, x), c(t, x), \mathbf{v}(t, x))^{T} \in \mathcal{B}_{R}$. We linearize the nonlinear equations (2.1) around fixed functions $(n(t, x), c(t, x), \mathbf{v}(t, x))^{T}$ to get the linearized equations on the unknown variables $(\Gamma(t, x), \Lambda(t, x), \mathbf{h}(t, x))^{T}$ with an external force 
$\left(f_{1}(t, x), f_{2}(t, x), \mathbf{g}(t, x)\right)^{T}$ as follows:

$$
\left\{\begin{array}{l}
\Gamma_{t}-\Delta \Gamma+(2 \mu n-\sigma) \Gamma+(\mathbf{v}+\overline{\mathbf{u}}) \cdot \nabla \Gamma+\mathbf{h} \cdot \nabla n \\
\quad+\chi \nabla \cdot[\Gamma \nabla c+n \nabla \Lambda]=f_{1}(t, x), \\
\Lambda_{t}-\Delta \Lambda+\Lambda+(\mathbf{v}+\overline{\mathbf{u}}) \cdot \nabla \Lambda+\mathbf{h} \cdot \nabla c-\Gamma=f_{2}(t, x), \\
\mathbf{h}_{t}-v \Delta \mathbf{h}+\mathbf{h} \cdot \nabla(\overline{\mathbf{u}}+\mathbf{v})+(\overline{\mathbf{u}}+\mathbf{v}) \cdot \nabla \mathbf{h}-\left(\mathcal{F}_{\mathbf{v}} \nabla \bar{P}\right) \mathbf{h} \\
\quad-\left(\mathcal{F}_{n} \nabla \bar{P}\right) \Gamma-\Gamma \nabla \Phi=\mathbf{g}(t, x), \\
\nabla \cdot \mathbf{h}=0, \quad \forall(t, x) \in\left(0, T^{*}\right) \times \Omega_{t},
\end{array}\right.
$$

where $\mathcal{F}_{\mathbf{v}}$ denotes the Fréchet derivative on $\mathbf{v}$ and $\mathbf{h}(t, x)=\left(h_{1}(t, x), h_{2}(t, x), h_{3}(t, x)\right)^{T}$.

In order to get some suitable prior estimates, we rewrite the linearized equations (2.5) as a coupled system,

$$
\begin{aligned}
& \Gamma_{t}-\Delta \Gamma+(2 \mu n-\sigma) \Gamma+(\mathbf{v}+\overline{\mathbf{u}}) \cdot \nabla \Gamma+\mathbf{h} \cdot \nabla n+\chi \nabla \cdot[\Gamma \nabla c+n \nabla \Lambda]=f_{1}(t, x), \\
& \Lambda_{t}-\Delta \Lambda+\Lambda+(\mathbf{v}+\overline{\mathbf{u}}) \cdot \nabla \Lambda+\mathbf{h} \cdot \nabla c-\Gamma=f_{2}(t, x), \\
& \partial_{t} h_{1}-v \Delta h_{1}+\frac{a}{T^{*}-t} h_{1}+k\left(T^{*}-t\right)^{2 a} h_{2}+\left(\frac{a x_{1}}{T^{*}-t}+k x_{2}\left(T^{*}-t\right)^{2 a}\right) \partial_{x_{1}} h_{1} \\
& \quad+\left(\frac{a x_{2}}{T^{*}-t}+k x_{1}\left(T^{*}-t\right)^{2 a}\right) \partial_{x_{2}} h_{1}-\frac{2 a x_{3}}{T^{*}-t} \partial_{x_{3}} h_{1}+h_{1} \partial_{x_{1}} w_{1}+w_{1} \partial_{x_{1}} h_{1} \\
& \quad+h_{2} \partial_{x_{2}} w_{1}+w_{2} \partial_{x_{2}} h_{1}+h_{3} \partial_{x_{3}} w_{1}+w_{3} \partial_{x_{3}} h_{1}-\Gamma \partial_{x_{1}} \Phi=\partial_{x_{1}} f(t, x)+g_{1}(t, x), \\
& \partial_{t} h_{2}-v \Delta h_{2}-k\left(T^{*}-t\right)^{2 a} h_{1}+\frac{a}{T^{*}-t} h_{2}+\left(\frac{a x_{1}}{T^{*}-t}+k x_{2}\left(T^{*}-t\right)^{2 a}\right) \partial_{x_{1}} h_{2} \\
& \quad+\left(\frac{a x_{2}}{T^{*}-t}-k x_{1}\left(T^{*}-t\right)^{2 a}\right) \partial_{x_{2}} h_{2}-\frac{2 a x_{3}}{T^{*}-t} \partial_{x_{3}} h_{2}+h_{1} \partial_{x_{1}} w_{2}+w_{1} \partial_{x_{1}} h_{2} \\
& \quad+h_{2} \partial_{x_{2}} w_{2}+w_{2} \partial_{x_{2}} h_{2}+h_{3} \partial_{x_{3}} w_{2}+w_{3} \partial_{x_{3}} h_{2}-\Gamma \partial_{x_{2}} \Phi=\partial_{x_{2}} f(t, x)+g_{2}(t, x), \\
& \partial_{t} h_{3}-v \Delta h_{3}-\frac{a}{T^{*}-t} h_{3}+\left(\frac{a x_{1}}{T^{*}-t}+k x_{2}\left(T^{*}-t\right)^{2 a}\right) \partial_{x_{1}} h_{3} \\
& \quad+\left(\frac{a x_{2}}{T^{*}-t}-k x_{1}\left(T^{*}-t\right)^{2 a}\right) \partial_{x_{2}} h_{3}-\frac{2 a x_{3}}{T^{*}-t} \partial_{x_{3}} h_{3}+h_{1} \partial_{x_{1}} w_{3}+w_{1} \partial_{x_{1}} h_{3} \\
& +h_{2} \partial_{x_{2}} w_{3}+w_{2} \partial_{x_{2}} h_{3}+h_{3} \partial_{x_{3}} w_{3}+w_{3} \partial_{x_{3}} h_{3}-\Gamma \partial_{x_{3}} \Phi=\partial_{x_{3}} f(t, x)+g_{3}(t, x),
\end{aligned}
$$

with the incompressibility condition

$$
\nabla \cdot \mathbf{h}=0
$$

where

$$
\begin{aligned}
f(t, x)= & -2 \Delta^{-1}\left[\sum_{i=1}^{3}\left(\partial_{x_{i}} w_{i} \partial_{x_{i}} h_{i}-\partial_{x_{i}}\left(\Gamma \partial_{x_{i}} \Phi\right)\right)+k\left(T^{*}-t\right)^{2 a}\left(\partial_{x_{1}} h_{2}-\partial_{x_{2}} h_{1}\right)\right. \\
& +\partial_{x_{2}} h_{1} \partial_{x_{1}} w_{2}+\partial_{x_{1}} h_{2} \partial_{x_{2}} w_{1}+\partial_{x_{3}} w_{1} \partial_{x_{1}} h_{3}+\partial_{x_{3}} h_{1} \partial_{x_{1}} w_{3} \\
& \left.+\partial_{x_{3}} w_{2} \partial_{x_{2}} h_{3}+\partial_{x_{3}} h_{2} \partial_{x_{2}} w_{3}\right]
\end{aligned}
$$


We introduce the similarity coordinates

$$
\begin{aligned}
& \tau=-\ln \left(T^{*}-t\right)+\ln T^{*}, \\
& y=\frac{x}{\sqrt{T^{*}-t}}
\end{aligned}
$$

where one can see the blowup time $T^{*}>0$ has been transformed into $+\infty$ in the similarity coordinates (2.12). So the local existence of linearized coupled system (2.6)-(2.10) with the incompressibility condition in some Sobolev space is equivalent to the global existence of linearized coupled system (2.13)-(2.17) in a related Sobolev space. This means the key point is to get the decay in time of solutions for system (2.13)-(2.17).

The linearized coupled system (2.6)-(2.10) under these coordinates is transformed into

$$
\begin{aligned}
& \partial_{\tau} \Gamma-\triangle_{y} \Gamma-\frac{y}{2} \cdot \nabla_{y} \Gamma+T^{*} e^{-\tau}(2 \mu n-\sigma) \Gamma+a y_{1} \partial_{y_{1}} \Gamma+a y_{2} \partial_{y_{2}} \Gamma-2 a y_{3} \partial_{y_{3}} \Gamma \\
& +k\left(T^{*}\right)^{2 a+1} e^{-(2 a+1) \tau}\left(y_{2} \partial_{y_{1}} \Gamma+y_{1} \partial_{y_{2}} \Gamma\right)+\left(T^{*}\right)^{\frac{1}{2}} e^{-\frac{1}{2} \tau} \mathbf{h} \cdot \nabla_{y} n+\chi \sum_{i=1}^{3} \partial_{y_{i}}\left(\Gamma \partial_{y_{i}} c\right) \\
& +\chi \nabla_{y} \cdot\left(n \nabla_{y} \Lambda\right)=T^{*} e^{-\tau} f_{1}\left(T^{*}\left(1-e^{-\tau}\right),\left(T^{*}\right)^{\frac{1}{2}} e^{-\frac{1}{2} \tau} y\right) \\
& \partial_{\tau} \Lambda-\triangle_{y} \Lambda-\frac{y}{2} \cdot \nabla_{y} \Lambda+T^{*} e^{-\tau} \Lambda+a y_{1} \partial_{y_{1}} \Lambda+a y_{2} \partial_{y_{2}} \Lambda-2 a y_{3} \partial_{y_{3}} \Lambda \\
& +k\left(T^{*}\right)^{2 a+1} e^{-(2 a+1) \tau}\left(y_{2} \partial_{y_{1}} \Lambda+y_{1} \partial_{y_{2}} \Lambda\right)+\left(T^{*}\right)^{\frac{1}{2}} e^{-\frac{1}{2} \tau}\left(\sum_{i=1}^{2} h_{i} \partial_{y_{i}} c_{i}-\Gamma\right) \\
& =T^{*} e^{-\tau} f_{2}\left(T^{*}\left(1-e^{-\tau}\right),\left(T^{*}\right)^{\frac{1}{2}} e^{-\frac{1}{2} \tau} y\right), \\
& \partial_{\tau} h_{1}-v \triangle_{y} h_{1}-\frac{y}{2} \cdot \nabla_{y} h_{1}+a h_{1}+a \partial_{y_{1}} h_{1}+a y_{2} \partial_{y_{2}} h_{1}-2 a y_{3} \partial_{y_{3}} h_{1} \\
& +k\left(T^{*}\right)^{2 a+1} e^{-(2 a+1) \tau}\left(h_{2}+y_{2} \partial_{y_{1}} h_{1}+y_{1} \partial_{y_{2}} h_{1}\right)-\left(T^{*}\right)^{\frac{1}{2}} e^{-\frac{1}{2} \tau} \Gamma \partial_{y_{1}} \Phi \\
& +\left(T^{*}\right)^{\frac{1}{2}} e^{-\frac{1}{2} \tau} \sum_{i=1}^{3}\left(h_{i} \partial_{y_{i}} w_{1}+w_{i} \partial_{y_{i}} h_{1}\right) \\
& =\left(T^{*}\right)^{\frac{1}{2}} \partial_{y_{1}} \bar{f}+T^{*} e^{-\tau} g_{1}\left(T^{*}\left(1-e^{-\tau}\right),\left(T^{*}\right)^{\frac{1}{2}} e^{-\frac{1}{2} \tau} y\right), \\
& \partial_{\tau} h_{2}-v \triangle_{y} h_{2}-\frac{y}{2} \cdot \nabla_{y} h_{2}+a h_{2}+a y_{1} \partial_{y_{1}} h_{2}+a y_{2} \partial_{y_{2}} h_{2}-2 a y_{3} \partial_{y_{3}} h_{2} \\
& +k\left(T^{*}\right)^{2 a+1} e^{-(2 a+1) \tau}\left(-h_{1}+y_{2} \partial_{y_{1}} h_{2}-y_{1} \partial_{y_{2}} h_{2}\right)-\left(T^{*}\right)^{\frac{1}{2}} e^{-\frac{1}{2} \tau} \Gamma \partial_{y_{2}} \Phi \\
& +\left(T^{*}\right)^{\frac{1}{2}} e^{-\frac{1}{2} \tau} \sum_{i=1}^{3}\left(h_{i} \partial_{y_{i}} w_{2}+w_{i} \partial_{y_{i}} h_{2}\right) \\
& =\left(T^{*}\right)^{\frac{1}{2}} \partial_{y_{2}} \bar{f}+T^{*} e^{-\tau} g_{2}\left(T^{*}\left(1-e^{-\tau}\right),\left(T^{*}\right)^{\frac{1}{2}} e^{-\frac{1}{2} \tau} y\right), \\
& \partial_{\tau} h_{3}-v \triangle_{y} h_{3}-\frac{y}{2} \cdot \nabla_{y} h_{3}-h_{3}+a y_{1} \partial_{y_{1}} h_{3}+a y_{2} \partial_{y_{2}} h_{3}-2 a y_{3} \partial_{y_{3}} h_{3} \\
& +k\left(T^{*}\right)^{2 a+1} e^{-(2 a+1) \tau}\left(y_{2} \partial_{y_{1}} h_{3}-y_{1} \partial_{y_{2}} h_{3}\right)-\left(T^{*}\right)^{\frac{1}{2}} e^{-\frac{1}{2} \tau} \Gamma \partial_{y_{3}} \Phi
\end{aligned}
$$




$$
\begin{gathered}
+\left(T^{*}\right)^{\frac{1}{2}} e^{-\frac{1}{2} \tau} \sum_{i=1}^{3}\left(h_{i} \partial_{y_{i}} w_{3}+w_{i} \partial_{y_{i}} h_{3}\right) \\
=\left(T^{*}\right)^{\frac{1}{2}} \partial_{y_{3}} \bar{f}+T^{*} e^{-\tau} g_{3}\left(T^{*}\left(1-e^{-\tau}\right),\left(T^{*}\right)^{\frac{1}{2}} e^{-\frac{1}{2} \tau} y\right),
\end{gathered}
$$

with the incompressibility condition

$$
\nabla_{y} \cdot \mathbf{h}=0
$$

where

$$
\begin{aligned}
\bar{f}= & -2 \triangle_{y}^{-1}\left[\sum_{i=1}^{3}\left(\partial_{y_{i}} w_{i} \partial_{y_{i}} h_{i}-\partial_{y_{i}}\left(\Gamma \partial_{y_{i}} \Phi\right)\right)+k\left(T^{*}\right)^{2 a} e^{-2 a \tau}\left(\partial_{y_{1}} h_{2}-\partial_{y_{2}} h_{1}\right)\right. \\
& +\partial_{y_{2}} h_{1} \partial_{y_{1}} w_{2}+\partial_{y_{1}} h_{2} \partial_{y_{2}} w_{1}+\partial_{y_{3}} w_{1} \partial_{y_{1}} h_{3}+\partial_{y_{3}} h_{1} \partial_{y_{1}} w_{3} \\
& \left.+\partial_{y_{3}} w_{2} \partial_{y_{2}} h_{3}+\partial_{y_{3}} h_{2} \partial_{y_{2}} w_{3}\right]
\end{aligned}
$$

We supplement the linearized system (2.13)-(2.17) with the initial data

$$
\left\{\begin{array}{l}
\Gamma(0, y)=\Gamma_{0}(y) \in H^{s}\left(\mathbb{R}^{3}\right) \\
\Lambda(0, y)=\Lambda_{0}(y) \in H^{s}\left(\mathbb{R}^{3}\right) \\
\mathbf{h}(0, y)=\mathbf{h}_{0}(y) \in H^{s}\left(\mathbb{R}^{3}\right)
\end{array}\right.
$$

and the boundary condition

$$
\left\{\begin{array}{l}
\lim _{|y| \rightarrow \infty} \Gamma(\tau, y)=0 \\
\lim _{|y| \rightarrow \infty} \Lambda(\tau, y)=0 \\
\lim _{|y| \rightarrow \infty} \mathbf{h}(\tau, y)=0
\end{array}\right.
$$

We first derive prior estimates of the linearized coupled system (2.13)-(2.17) with the initial data (2.19) and condition (2.20).

Lemma 2.1 Let $s>0,0<a \ll \frac{1}{8}$ and $T^{*} \in(0,1)$ be constants. Assume that $\|\Phi\|_{\mathbb{H}^{S^{s+3}}\left(\mathbb{R}^{3}\right)} \lesssim$ $R \ll 1, f_{i} \in \mathbb{C}^{1}\left((0,+\infty), \mathbb{H}^{s}\left(\mathbb{R}^{3}\right)\right)(i=1,2), \boldsymbol{g} \in \mathbb{C}^{1}\left((0,+\infty), H^{s}\left(\mathbb{R}^{3}\right)\right)$ and $(n, c, v)^{T} \in \mathcal{B}_{R}$. Then, for any $\tau>0$, the solution $(\Gamma, \Lambda, \boldsymbol{h})^{T}$ of linearized coupled system (2.13)-(2.17) with the initial data (2.19) and condition (2.20) satisfies

$$
\begin{aligned}
\int_{\mathbb{R}^{3}}\left(|\Gamma|^{2}+|\Lambda|^{2}+|\boldsymbol{h}|^{2}\right) d y \lesssim & e^{-C \tau}\left(\int_{\mathbb{R}^{3}}\left(\left|\Gamma_{0}\right|^{2}+\left|\Lambda_{0}\right|^{2}+\left|\boldsymbol{h}_{0}\right|^{2}\right) d y\right. \\
& \left.+\int_{0}^{+\infty} \int_{\mathbb{R}^{3}}\left(\left|f_{1}\right|^{2}+\left|f_{2}\right|^{2}+|\boldsymbol{g}|^{2}\right) d y d \tau\right),
\end{aligned}
$$

where $C$ is a positive constant. 
Proof Multiplying both sides of (2.13)-(2.17) by $\Gamma, \Lambda, h_{1}, h_{2}$ and $h_{3}$, respectively, then integrating by parts (using the boundary condition (2.20)), we have

$$
\begin{aligned}
& \frac{1}{2} \frac{d}{d \tau}\|\Gamma\|_{\mathbb{L}^{2}}^{2}+\left\|\nabla_{y} \Gamma\right\|_{\mathbb{L}^{2}}^{2}+\left(\frac{3}{4}-T^{*} e^{-\tau} \sigma\right)\|\Gamma\|_{\mathbb{L}^{2}}^{2}+2 \mu T^{*} e^{-\tau} \int_{\mathbb{R}^{3}} n \Gamma^{2} d y \\
& +\chi \sum_{i=1}^{3} \int_{\mathbb{R}^{3}}\left(\Gamma \partial_{y_{i}} c \partial_{y_{i}} \Gamma+n \partial_{y_{i}}^{2} \Lambda\right) d y+\left(T^{*}\right)^{\frac{1}{2}} e^{-\frac{1}{2} \tau} \int_{\mathbb{R}^{3}}\left(\mathbf{h} \cdot \nabla_{y} n\right) \Gamma d y \\
& =T^{*} e^{-\tau} \int_{\mathbb{R}^{3}} \Gamma f_{1} d y, \\
& \frac{1}{2} \frac{d}{d \tau}\|\Lambda\|_{\mathbb{L}^{2}}^{2}+\left\|\nabla_{y} \Lambda\right\|_{\mathbb{L}^{2}}^{2}+\left(\frac{3}{4}+T^{*} e^{-\tau}\right)\|\Lambda\|_{\mathbb{L}^{2}}^{2} \\
& +\left(T^{*}\right)^{\frac{1}{2}} e^{-\frac{1}{2} \tau} \int_{\mathbb{R}^{3}}\left(\sum_{i=1}^{2} h_{i} \partial_{y_{i}} c_{i}-\Gamma\right) \Lambda d y=T^{*} e^{-\tau} \int_{\mathbb{R}^{3}} \Lambda f_{2} d y, \\
& \frac{1}{2} \frac{d}{d \tau}\left\|h_{1}\right\|_{\mathbb{L}^{2}}^{2}+v \sum_{i, j=1}^{3}\left\|\partial_{y_{i}} h_{j}\right\|_{\mathbb{L}^{2}}^{2}+\left(a+\frac{3}{4}\right)\left\|h_{1}\right\|_{\mathbb{L}^{2}}^{2}+k\left(T^{*}\right)^{2 a+1} e^{-2(a+1) \tau} \int_{\mathbb{R}^{3}} h_{1} h_{2} d y \\
& +\left(T^{*}\right)^{\frac{1}{2}} e^{-\frac{1}{2} \tau} \sum_{i=1}^{3} \int_{\mathbb{R}^{3}} h_{1}\left(h_{i} \partial_{y_{i}} w_{1}+w_{i} \partial_{y_{i}} h_{1}\right) d y \\
& -\left(T^{*}\right)^{\frac{1}{2}} e^{-\frac{1}{2} \tau} \int_{\mathbb{R}^{3}} \Gamma \partial_{y_{1}} \Phi h_{1} d y=\left(T^{*}\right)^{\frac{1}{2}} \int_{\mathbb{R}^{3}} h_{1} \partial_{y_{1}} \bar{f} d y+\int_{\mathbb{R}^{3}} h_{1} g_{1} d y, \\
& \frac{1}{2} \frac{d}{d \tau}\left\|h_{2}\right\|_{\mathbb{L}^{2}}^{2}+v \sum_{i, j=1}^{3}\left\|\partial_{y_{i}} h_{j}\right\|_{\mathbb{L}^{2}}^{2}+\left(a+\frac{3}{4}\right)\left\|h_{2}\right\|_{\mathbb{L}^{2}}^{2}-k\left(T^{*}\right)^{2 a+1} e^{-2(a+1) \tau} \int_{\mathbb{R}^{3}} h_{1} h_{2} d y \\
& +\left(T^{*}\right)^{\frac{1}{2}} e^{-\frac{1}{2} \tau} \sum_{i=1}^{3} \int_{\mathbb{R}^{3}} h_{2}\left(h_{i} \partial_{y_{i}} w_{2}+w_{i} \partial_{y_{i}} h_{2}\right) d y \\
& -\left(T^{*}\right)^{\frac{1}{2}} e^{-\frac{1}{2} \tau} \int_{\mathbb{R}^{3}} \Gamma \partial_{y_{2}} \Phi h_{2} d y=\left(T^{*}\right)^{\frac{1}{2}} \int_{\mathbb{R}^{3}} h_{2} \partial_{y_{2}} \bar{f} d y+\int_{\mathbb{R}^{3}} h_{2} g_{2} d y,
\end{aligned}
$$

and

$$
\begin{aligned}
\frac{1}{2} \frac{d}{d \tau} & \left\|h_{3}\right\|_{\mathbb{L}^{2}}^{2}+v \sum_{i, j=1}^{3}\left\|\partial_{y_{i}} h_{j}\right\|_{\mathbb{L}^{2}}^{2}+\left(\frac{3}{4}-2 a\right)\left\|h_{3}\right\|_{\mathbb{L}^{2}}^{2} \\
& +\left(T^{*}\right)^{\frac{1}{2}} e^{-\frac{1}{2} \tau} \sum_{i=1}^{3} \int_{\mathbb{R}^{3}} h_{3}\left(h_{i} \partial_{y_{i}} w_{3}+w_{i} \partial_{y_{i}} h_{3}\right) d y \\
& -\left(T^{*}\right)^{\frac{1}{2}} e^{-\frac{1}{2} \tau} \int_{\mathbb{R}^{3}} \Gamma \partial_{y_{3}} \Phi h_{3} d y=\left(T^{*}\right)^{\frac{1}{2}} \int_{\mathbb{R}^{3}} h_{3} \partial_{y_{3}} \bar{f} d y+\int_{\mathbb{R}^{3}} h_{3} g_{3} d y
\end{aligned}
$$

Summing up (2.21)-(2.25), then

$$
\begin{aligned}
& \frac{1}{2} \sum_{i=1}^{3} \frac{d}{d \tau}\left(\left\|h_{i}\right\|_{\mathbb{L}^{2}}^{2}+\|\Gamma\|_{\mathbb{L}^{2}}^{2}+\|\Lambda\|_{\mathbb{L}^{2}}^{2}\right)+\left\|\nabla_{y} \Gamma\right\|_{\mathbb{L}^{2}}^{2}+\left\|\nabla_{y} \Lambda\right\|_{\mathbb{L}^{2}}^{2}+3 \nu \sum_{i, j=1}^{3}\left\|\partial_{y_{i}} h_{j}\right\|_{\mathbb{L}^{2}}^{2} \\
& \quad+\left(\frac{3}{4}-T^{*} e^{-\tau} \sigma\right)\|\Gamma\|_{\mathbb{L}^{2}}^{2}+\left(\frac{3}{4}+T^{*} e^{-\tau}\right)\|\Lambda\|_{\mathbb{L}^{2}}^{2}+\left(a+\frac{3}{4}\right)\left(\left\|h_{1}\right\|_{\mathbb{L}^{2}}^{2}+\left\|h_{2}\right\|_{\mathbb{L}^{2}}^{2}\right)
\end{aligned}
$$




$$
\begin{aligned}
& +\left(\frac{3}{4}-2 a\right)\left\|h_{3}\right\|_{\mathbb{L}^{2}}^{2}+2 \mu T^{*} e^{-\tau} \int_{\mathbb{R}^{3}} n \Gamma^{2} d y-\chi \sum_{i=1}^{3} \int_{\mathbb{R}^{3}}\left(\Gamma \partial_{y_{i}} c \partial_{y_{i}} \Gamma+n \partial_{y_{i}}^{2} \Lambda\right) d y \\
& +\left(T^{*}\right)^{\frac{1}{2}} e^{-\frac{1}{2} \tau} \int_{\mathbb{R}^{3}}\left(\mathbf{h} \cdot \nabla_{y} n\right) \Gamma d y+\left(T^{*}\right)^{\frac{1}{2}} e^{-\frac{1}{2} \tau} \int_{\mathbb{R}^{3}}\left(\sum_{i=1}^{3} h_{i} \partial_{y_{i}} c_{i}-\Gamma\right) \Lambda d y \\
& +\left(T^{*}\right)^{\frac{1}{2}} e^{-\frac{1}{2} \tau} \sum_{i=1}^{3} \int_{\mathbb{R}^{3}} \Gamma \partial_{y_{i}} \Phi h_{i} d y+\left(T^{*}\right)^{\frac{1}{2}} e^{-\frac{1}{2} \tau} \sum_{i=1}^{3} \int_{\mathbb{R}^{3}} h_{1}\left(h_{i} \partial_{y_{i}} w_{1}+w_{i} \partial_{y_{i}} h_{1}\right) d y \\
& +\left(T^{*}\right)^{\frac{1}{2}} e^{-\frac{1}{2} \tau} \sum_{i=1}^{3} \int_{\mathbb{R}^{3}} h_{2}\left(h_{i} \partial_{y_{i}} w_{2}+w_{i} \partial_{y_{i}} h_{2}\right) d y \\
& +\left(T^{*}\right)^{\frac{1}{2}} e^{-\frac{1}{2} \tau} \sum_{i=1}^{3} \int_{\mathbb{R}^{3}} h_{3}\left(h_{i} \partial_{y_{i}} w_{3}+w_{i} \partial_{y_{i}} h_{3}\right) d y \\
& =\left(T^{*}\right)^{\frac{1}{2}} \sum_{i=1}^{3} \int_{\mathbb{R}^{3}} h_{i} \partial_{y_{i}} \bar{f} d y+T^{*} e^{-\tau} \int_{\mathbb{R}^{3}}\left(\Gamma f_{1}+\Lambda f_{2}+\sum_{i=1}^{3} h_{i} g_{i}\right) d y, \quad \forall \tau>0 .
\end{aligned}
$$

We now estimate each coupled nonlinear term in (2.26). Note that $(n, c, \mathbf{v})^{T} \in \mathcal{B}_{R}$ and $H^{\frac{5}{2}}\left(\mathbb{R}^{3}\right) \subset L^{\infty}\left(\mathbb{R}^{3}\right)$. We use Young's inequality to derive

$$
\begin{aligned}
& \left|2 \mu T^{*} e^{-\tau} \int_{\mathbb{R}^{3}} n \Gamma^{2} d y\right| \lesssim C_{R}\|\Gamma\|_{\mathbb{L}^{2}}^{2}, \\
& \left|\sum_{i=1}^{3} \int_{\mathbb{R}^{3}}\left(\Gamma \partial_{y_{i}} c \partial_{y_{i}} \Gamma+n \partial_{y_{i}}^{2} \Lambda\right) d y\right| \lesssim C_{R}\left(\|\Gamma\|_{\mathbb{L}^{2}}^{2}+\sum_{i=1}^{3}\left(\left\|\partial_{y_{i}} \Gamma\right\|_{\mathbb{L}^{2}}^{2}+\left\|\partial_{y_{i}} \Lambda\right\|_{\mathbb{L}^{2}}^{2}\right)\right) \\
& \left|\left(T^{*}\right)^{\frac{1}{2}} e^{-\frac{1}{2} \tau} \int_{\mathbb{R}^{3}}\left(\mathbf{h} \cdot \nabla_{y} n\right) \Gamma d y\right| \lesssim C_{R}\left(\|\Gamma\|_{\mathbb{L}^{2}}^{2}+\sum_{i=1}^{3}\left\|h_{i}\right\|_{\mathbb{L}^{2}}^{2}\right), \\
& \left|\left(T^{*}\right)^{\frac{1}{2}} e^{-\frac{1}{2} \tau} \int_{\mathbb{R}^{3}}\left(\sum_{i=1}^{3} h_{i} \partial_{y_{i}} d_{i}-\Gamma\right) \Lambda d y\right| \\
& \quad \frac{\left(T^{*}\right)^{\frac{1}{2}}}{2} e^{-\frac{1}{2} \tau}\left(2\|\Lambda\|_{\mathbb{L}^{2}}^{2}+\|\Gamma\|_{\mathbb{L}^{2}}^{2}\right)+C_{R} \sum_{i=1}^{3}\left\|h_{i}\right\|_{\mathbb{L}^{2}}^{2}, \\
& \left|\left(T^{*}\right)^{\frac{1}{2}} e^{-\frac{1}{2} \tau} \sum_{i=1}^{3} \int_{\mathbb{R}^{3}} \Gamma \partial_{y_{i}} \Phi h_{i} d y\right| \lesssim C_{R}\left(\|\Gamma\|_{\mathbb{L}^{2}}^{2}+\sum_{i=1}^{3}\left\|h_{i}\right\|_{\mathbb{L}^{2}}^{2}\right),
\end{aligned}
$$

and

$$
\begin{aligned}
&\left|\sum_{i=1}^{3} \int_{\mathbb{R}^{3}} h_{1}\left(h_{i} \partial_{x_{i}} w_{1}+w_{i} \partial_{y_{i}} h_{1}\right) d y\right| \lesssim C_{R} \sum_{i=1}^{3}\left(\left\|h_{i}\right\|_{\mathbb{L}^{2}}^{2}+\left\|\partial_{y_{i}} h_{1}\right\|_{\mathbb{L}^{2}}\right), \\
&\left|\sum_{i=1}^{3} \int_{\mathbb{R}^{3}} h_{2}\left(h_{i} \partial_{y_{i}} w_{2}+w_{i} \partial_{y_{i}} h_{2}\right) d y\right| \lesssim C_{R} \sum_{i=1}^{3}\left(\left\|h_{i}\right\|_{\mathbb{L}^{2}}^{2}+\left\|\partial_{y_{i}} h_{2}\right\|_{\mathbb{L}^{2}}\right), \\
&\left|\sum_{i=1}^{3} \int_{\mathbb{R}^{3}} h_{3}\left(h_{i} \partial_{y_{i}} w_{3}+w_{i} \partial_{y_{i}} h_{3}\right) d y\right| \lesssim C_{R} \sum_{i=1}^{3}\left(\left\|h_{i}\right\|_{\mathbb{L}^{2}}^{2}+\left\|\partial_{y_{i}} h_{3}\right\|_{\mathbb{L}^{2}}\right),
\end{aligned}
$$


where $C_{R}, C_{a, R}, C_{\kappa, R}$ are three positive constants depending on $R, a, R$ and $\kappa, R$, respectively.

On the other hand, by (2.18) and the standard Calderon-Zygmund theory, i.e. for the Riesz operator $\mathcal{R}$, we have $\|\mathcal{R} w\|_{\mathbb{L}^{p}} \leq\|w\|_{\mathbb{L}^{p}}$ with $1<p<\infty$, we also use Young's inequality to get

$$
\left|\sum_{i=1}^{3} \int_{\Omega} h_{i} \partial_{y_{i}} \bar{f} d y\right| \lesssim C_{R} \sum_{i, j=1}^{3}\left(\|\Gamma\|_{\mathbb{L}^{2}}^{2}+\left\|h_{i}\right\|_{\mathbb{L}^{2}}^{2}+\left\|\partial_{y_{i}} h_{j}\right\|_{\mathbb{L}^{2}}^{2}\right)
$$

and

$$
\begin{aligned}
\left|T^{*} e^{-\tau} \int_{\Omega}\left(\Gamma f_{1}+\Lambda f_{2}+\sum_{i=1}^{3} h_{i} g_{i}\right) d y\right| \\
\leq T^{*} e^{-\tau}\left[b\left(\|\Gamma\|_{\mathbb{L}^{2}}^{2}+\|\Lambda\|_{\mathbb{L}^{2}}^{2}\right)+b^{-1}\left(\left\|f_{1}\right\|_{\mathbb{L}^{2}}^{2}+\left\|f_{2}\right\|_{\mathbb{L}^{2}}^{2}\right)\right. \\
\left.\quad+\sum_{i=1}^{3}\left(b\left\|h_{i}\right\|_{\mathbb{L}^{2}}^{2}+b^{-1}\left\|g_{i}\right\|_{\mathbb{L}^{2}}^{2}\right)\right],
\end{aligned}
$$

where the positive constant $b<1$.

Thus by (2.27)-(2.30), it follows from (2.26) that

$$
\begin{aligned}
\frac{1}{2} \sum_{i=1}^{3} & \frac{d}{d \tau}\left\|h_{i}\right\|_{\mathbb{L}^{2}}^{2}+\left(1-C_{R}\right)\left\|\nabla_{y} \Gamma\right\|_{\mathbb{L}^{2}}^{2}+\left(1-C_{R}\right)\left\|\nabla_{y} \Lambda\right\|_{\mathbb{L}^{2}}^{2}+\left(3 v-C_{R}\right) \sum_{i, j=1}^{3}\left\|\partial_{y_{i}} h_{j}\right\|_{\mathbb{L}^{2}}^{2} \\
& +\left(\frac{3}{4}-T^{*} e^{-\tau}(b+\sigma)-\frac{\left(T^{*}\right)^{\frac{1}{2}}}{2} e^{-\frac{1}{2} \tau}-C_{R}\right)\|\Gamma\|_{\mathbb{L}^{2}}^{2} \\
& +\left(\frac{3}{4}+T^{*} e^{-\tau}(1-b)-\left(T^{*}\right)^{\frac{1}{2}} e^{-\frac{1}{2} \tau}-C_{R}\right)\|\Lambda\|_{\mathbb{L}^{2}}^{2} \\
& +\left(a+\frac{3}{4}-T^{*} e^{-\tau} b-C_{R}\right)\left(\left\|h_{1}\right\|_{\mathbb{L}^{2}}^{2}+\left\|h_{2}\right\|_{\mathbb{L}^{2}}^{2}\right)+\left(\frac{3}{4}-2 a-T^{*} e^{-\tau} b-C_{R}\right)\left\|h_{3}\right\|_{\mathbb{L}^{2}}^{2} \\
\lesssim & b^{-1}\left(\left\|f_{1}\right\|_{\mathbb{L}^{2}}^{2}+\left\|f_{2}\right\|_{\mathbb{L}^{2}}^{2}+\sum_{i=1}^{3}\left\|g_{i}\right\|_{\mathbb{L}^{2}}^{2}\right)
\end{aligned}
$$

where $C_{R}$ is a positive constant depending on $R$, which can be very small if constants $R$ is small.

There exists a sufficiently small positive constant $b \in(0,1)$ such that

$$
\begin{aligned}
& 1-C_{R}>0, \quad 1-C_{R}>0, \quad 3 v-C_{R}>0, \\
& \frac{3}{4}-T^{*} e^{-\tau}(b+\sigma)-\frac{\left(T^{*}\right)^{\frac{1}{2}}}{2} e^{-\frac{1}{2} \tau}-C_{R}>0, \\
& \frac{3}{4}+T^{*} e^{-\tau}(1-b)-\left(T^{*}\right)^{\frac{1}{2}} e^{-\frac{1}{2} \tau}-C_{R}>0, \\
& a+\frac{3}{4}-T^{*} e^{-\tau} b-C_{R}>0, \quad \frac{3}{4}-2 a-T^{*} e^{-\tau} b-C_{R}>0 .
\end{aligned}
$$


Hence, applying Gronwall's inequality to (2.31), there exists a positive constant $C$ such that

$$
\begin{aligned}
\|\Gamma\|_{\mathbb{L}^{2}}^{2}+\|\Gamma\|_{\mathbb{L}^{2}}^{2}+\sum_{i=1}^{3}\left\|h_{i}\right\|_{\mathbb{L}^{2}}^{2} \lesssim & e^{-C \tau} \int_{\mathbb{R}^{3}}\left(\Gamma_{0}^{2}+\Lambda_{0}^{2}+\sum_{i=1}^{3} h_{0 i}^{2}\right) d y \\
& +e^{-C \tau} \int_{0}^{+\infty} \int_{\mathbb{R}^{3}}\left(f_{1}^{2}+f_{2}^{2}+\sum_{i=1}^{3} g_{i}^{2} d \tau\right) d y, \quad \forall \tau>0 .
\end{aligned}
$$

In what follows, we plan to carry out higher order derivative estimates to the solutions of linearized system (2.8)-(2.10). For a fixed constant $s>0$, applying $\nabla^{s}=\partial_{y_{i}}^{s}$ to both sides of (2.22)-(2.10), we obtain

$$
\begin{aligned}
& \partial_{\tau} \nabla_{y}^{s} \Gamma-\triangle_{y} \nabla_{y}^{s} \Gamma-\frac{y}{2} \cdot \nabla_{y}^{s+1} \Gamma+\left(T^{*} e^{-\tau}(2 \mu n-\sigma)-\frac{s}{2}\right) \nabla_{y}^{s} \Gamma+a y_{1} \partial_{y_{1}} \nabla_{y}^{s} \Gamma+a y_{2} \partial_{y_{2}} \nabla_{y}^{s} \Gamma \\
& -2 a y_{3} \partial_{y_{3}} \nabla_{y}^{s} \Gamma+k\left(T^{*}\right)^{2 a+1} e^{-(2 a+1) \tau}\left(2 s \nabla_{y}^{s} \Gamma+y_{2} \partial_{y_{1}} \nabla_{y}^{s} \Gamma+y_{1} \partial_{y_{2}} \nabla_{y}^{s} \Gamma\right) \\
& +\left(T^{*}\right)^{\frac{1}{2}} e^{-\frac{1}{2} \tau} \nabla_{y}^{s}\left(\mathbf{h} \cdot \nabla_{y} n\right)+\chi \sum_{i=1}^{3} \partial_{y_{i}} \nabla_{y}^{s}\left(\Gamma \partial_{y_{i}} c\right)+\chi \nabla_{y}^{s}\left(\nabla_{y} \cdot\left(n \nabla_{y} \Lambda\right)\right) \\
& =T^{*} e^{-\tau} \nabla_{y}^{s} f_{1}, \\
& \partial_{\tau} \nabla_{y}^{s} \Lambda-\triangle_{y} \nabla_{y}^{s} \Lambda-\frac{y}{2} \cdot \nabla_{y}^{s+1} \Lambda+\left(T^{*} e^{-\tau}-\frac{s}{2}\right) \nabla_{y}^{s} \Lambda+a y_{1} \partial_{y_{1}} \nabla_{y}^{s} \Lambda+a y_{2} \partial_{y_{2}} \nabla_{y}^{s} \Lambda \\
& -2 a y_{3} \partial_{y_{3}} \nabla_{y}^{s} \Lambda+k\left(T^{*}\right)^{2 a+1} e^{-(2 a+1) \tau}\left(2 s \nabla_{y}^{s} \Lambda+y_{2} \partial_{y_{1}} \nabla_{y}^{s} \Lambda+y_{1} \partial_{y_{2}} \nabla_{y}^{s} \Lambda\right) \\
& +\left(T^{*}\right)^{\frac{1}{2}} e^{-\frac{1}{2} \tau}\left(\sum_{i=1}^{2} \nabla_{y}^{s}\left(h_{i} \partial_{y_{i}} c_{i}\right)-\Gamma\right)=T^{*} e^{-\tau} \nabla_{y}^{s} f_{2}, \\
& \partial_{\tau} \nabla_{y}^{s} h_{1}-v \triangle_{y} \nabla_{y}^{s} h_{1}-\frac{y}{2} \cdot \partial_{y} \nabla_{y}^{s} h_{1}+\left(a-\frac{s}{2}\right) \nabla_{y}^{s} h_{1}+a y_{1} \partial_{y_{1}} \nabla_{y}^{s} h_{1}+a y_{2} \partial_{y_{2}} \nabla_{y}^{s} h_{1} \\
& -2 a y_{3} \partial_{y_{3}} \nabla_{y}^{s} h_{1}+k\left(T^{*}\right)^{2 a+1} e^{-(2 a+1) \tau}\left(\nabla_{y}^{s} h_{2}+2 s \nabla_{y}^{s} h_{1}+y_{2} \partial_{y_{1}} \nabla_{y}^{s} h_{1}+y_{1} \partial_{y_{2}} \nabla_{y}^{s} h_{1}\right) \\
& -\left(T^{*}\right)^{\frac{1}{2}} e^{-\frac{1}{2} \tau} \nabla_{y}^{s}\left(\Gamma \partial_{y_{1}} \Phi\right)+\left(T^{*}\right)^{\frac{1}{2}} e^{-\frac{1}{2} \tau} \sum_{i=1}^{3}\left(\nabla_{y}^{s} h_{i} \partial_{y_{i}} w_{1}+w_{i} \partial_{y_{i}} \nabla_{y}^{s} h_{1}\right)=\tilde{g}_{1}, \\
& \partial_{\tau} \nabla_{y}^{s} h_{2}-v \triangle_{y} \nabla_{y}^{s} h_{2}-\frac{y}{2} \cdot \partial_{y} \nabla_{y}^{s} h_{2}+\left(a-\frac{s}{2}\right) \nabla_{y}^{s} h_{2}+a y_{1} \partial_{y_{1}} \nabla_{y}^{s} h_{2}+a y_{2} \partial_{y_{2}} \nabla_{y}^{s} h_{2} \\
& -2 a y_{3} \partial_{y_{3}} \nabla_{y}^{s} h_{2}+k\left(T^{*}\right)^{2 a+1} e^{-(2 a+1) \tau}\left(-\nabla_{y}^{s} h_{1}+y_{2} \partial_{y_{1}} \nabla_{y}^{s} h_{2}-y_{1} \partial_{y_{2}} \nabla_{y}^{s} h_{2}\right) \\
& -\left(T^{*}\right)^{\frac{1}{2}} e^{-\frac{1}{2} \tau} \nabla_{y}^{s}\left(\Gamma \partial_{y_{2}} \Phi\right)+\left(T^{*}\right)^{\frac{1}{2}} e^{-\frac{1}{2} \tau} \sum_{i=1}^{3}\left(\nabla_{y}^{s} h_{i} \partial_{y_{i}} w_{2}+w_{i} \partial_{y_{i}} \nabla_{y}^{s} h_{2}\right)=\tilde{g}_{2}, \\
& \partial_{\tau} \nabla_{y}^{s} h_{3}-v \triangle_{y} \nabla_{y}^{s} h_{3}-\frac{y}{2} \cdot \partial_{y} \nabla_{y}^{s} h_{3}-\left(2 a+\frac{s}{2}\right) \nabla_{y}^{s} h_{3}+a y_{1} \partial_{y_{1}} \nabla_{y}^{s} h_{3}+a y_{2} \partial_{y_{2}} \nabla_{y}^{s} h_{3} \\
& -2 a y_{3} \partial_{y_{3}} \nabla_{y}^{s} h_{3}+k\left(T^{*}\right)^{2 a+1} e^{-(2 a+1) \tau}\left(y_{2} \partial_{y_{1}} \nabla_{y}^{s} h_{3}-y_{1} \partial_{y_{2}} \nabla_{y}^{s} h_{3}\right) \\
& +\left(T^{*}\right)^{\frac{1}{2}} e^{-\frac{1}{2} \tau} \sum_{i=1}^{3}\left(\nabla_{y}^{s} h_{i} \partial_{y_{i}} w_{3}+w_{i} \partial_{y_{i}} \nabla_{y}^{s} h_{3}\right)-\left(T^{*}\right)^{\frac{1}{2}} e^{-\frac{1}{2} \tau} \nabla_{y}^{s}\left(\Gamma \partial_{y_{3}} \Phi\right)=\tilde{g}_{3},
\end{aligned}
$$


where

$$
\begin{aligned}
& \tilde{g}_{1}:=\left(T^{*}\right)^{\frac{1}{2}} \partial_{y_{1}} \nabla_{y}^{s} \bar{f}+T^{*} e^{-\tau} \nabla_{y}^{s} g_{1} \\
& -\left(T^{*}\right)^{\frac{1}{2}} e^{-\frac{1}{2} \tau} \sum_{j=1}^{3} \sum_{i_{1}+i_{2}=s, 0 \leq i_{2} \leq s-1}\left(\nabla_{y}^{i_{2}} h_{j} \partial_{y_{j}} \nabla_{y}^{i_{1}} w_{1}+\nabla_{y}^{i_{1}} w_{j} \partial_{y_{j}} \nabla_{y}^{i_{2}} h_{1}\right), \\
& \tilde{g}_{2}:=\left(T^{*}\right)^{\frac{1}{2}} \partial_{y_{2}} \nabla_{y}^{s} \bar{f}+T^{*} e^{-\tau} \nabla_{y}^{s} g_{2} \\
& -\left(T^{*}\right)^{\frac{1}{2}} e^{-\frac{1}{2} \tau} \sum_{j=1}^{3} \sum_{i_{1}+i_{2}=s, 0 \leq i_{2} \leq s-1}\left(\nabla_{y}^{i_{2}} h_{j} \partial_{y_{j}} \nabla_{y}^{i_{1}} w_{2}+\nabla_{y}^{i_{1}} w_{j} \partial_{y_{j}} \nabla_{y}^{i_{2}} h_{2}\right), \\
& \tilde{g}_{3}:=\left(T^{*}\right)^{\frac{1}{2}} \partial_{y_{3}} \nabla_{y}^{s} \bar{f}+T^{*} e^{-\tau} \nabla_{y}^{s} g_{3} \\
& -\left(T^{*}\right)^{\frac{1}{2}} e^{-\frac{1}{2} \tau} \sum_{j=1}^{3} \sum_{i_{1}+i_{2}=s, 0 \leq i_{2} \leq s-1}\left(\nabla_{y}^{i_{2}} h_{j} \partial_{y_{j}} \nabla_{y}^{i_{1}} w_{3}+\nabla_{y}^{i_{1}} w_{j} \partial_{y_{j}} \nabla_{y}^{i_{2}} h_{3}\right) .
\end{aligned}
$$

We now have the following higher order derivatives estimates.

Lemma 2.2 Let $0<a \ll \frac{1}{8}$ and $0<s<\frac{3}{2}-5$ a be constants. Assume that $\|\Phi\|_{\mathbb{H}^{s+3}\left(\mathbb{R}^{3}\right)} \lesssim R \ll$ $1, f_{i} \in \mathbb{C}^{1}\left((0,+\infty), \mathbb{H}^{s}\left(\mathbb{R}^{3}\right)\right)(i=1,2), \boldsymbol{g} \in \mathbb{C}^{1}\left((0,+\infty), H^{s}\left(\mathbb{R}^{3}\right)\right)$ and $(n, c, \boldsymbol{v})^{T} \in \mathcal{B}_{R}$. Then, for any $\tau>0$, the solution $(\Gamma, \Lambda, \boldsymbol{h})^{T}$ of the linearized coupled system (2.13)-(2.17) with the initial data (2.19) and condition (2.20) satisfies

$$
\begin{aligned}
& \int_{\mathbb{R}^{3}}\left(\left|\nabla_{y}^{s} \Gamma\right|^{2}+\left|\nabla_{y}^{s} \Lambda\right|^{2}+\sum_{i=1}^{3}\left|\nabla_{y}^{s} h_{i}\right|^{2}\right) d y \\
& \lesssim e^{-C_{R, T^{*} \tau}} \int_{\mathbb{R}^{3}}\left(\left|\nabla_{y}^{s} \Gamma_{0}\right|^{2}+\left|\nabla_{y}^{s} \Lambda_{0}\right|^{2}+\sum_{i=1}^{3}\left|\nabla_{y}^{s} h_{0 i}\right|^{2}\right) d y \\
& \quad+e^{-C_{R, T^{*}} \tau} \int_{0}^{+\infty}\left(\left\|\nabla_{y}^{s} f_{1}\right\|_{\mathbb{L}^{2}}^{2}+\left\|\nabla_{y}^{s} f_{2}\right\|_{\mathbb{L}^{2}}^{2}+\sum_{i=1}^{3}\left\|\nabla_{y}^{s} g_{i}\right\|_{\mathbb{L}^{2}}^{2}\right) d \tau, \quad \forall \tau>0,
\end{aligned}
$$

where $C_{R, T^{*}}$ is a positive constant depending on constants $R, T^{*}$.

Proof Taking the inner product of both sides of (2.32)-(2.36) by $\nabla_{y}^{s} \Gamma, \nabla_{y}^{s} \Lambda, \nabla_{y}^{s} h_{1}, \nabla_{y}^{s} h_{2}$ and $\nabla_{y}^{s} h_{3}$, respectively, then integrating by parts, we have

$$
\begin{aligned}
\frac{1}{2} \frac{d}{d \tau} & \left\|\nabla_{y}^{s} \Gamma\right\|_{\mathbb{L}^{2}}^{2}+\left\|\nabla_{y}^{s+1} \Gamma\right\|_{\mathbb{L}^{2}}^{2}+\left(\frac{3}{4}-\frac{s}{2}-T^{*} e^{-\tau} \sigma++2 s \kappa\left(T^{*}\right)^{2 a+1} e^{-(2 a+1) \tau}\right)\left\|\nabla_{y}^{s} \Gamma\right\|_{\mathbb{L}^{2}}^{2} \\
& +2 \mu T^{*} e^{-\tau} \int_{\mathbb{R}^{3}} n\left|\nabla_{y} \Gamma\right|^{2} d y+\left(T^{*}\right)^{\frac{1}{2}} e^{-\frac{1}{2} \tau} \int_{\mathbb{R}^{3}} \nabla_{y}^{s}\left(\mathbf{h} \cdot \nabla_{y} n\right) \cdot \nabla_{y}^{s} \Gamma d y \\
& +\chi \sum_{i=1}^{3} \int_{\mathbb{R}^{3}} \partial_{y_{i}} \nabla_{y}^{s}\left(\Gamma \partial_{y_{i}} c\right) \cdot \nabla_{y}^{s} \Gamma d y+\chi \int_{\mathbb{R}^{3}} \nabla_{y}^{s}\left(\nabla_{y} \cdot\left(n \nabla_{y} \Lambda\right)\right) \cdot \nabla_{y}^{s} \Gamma d y \\
= & T^{*} e^{-\tau} \int_{\mathbb{R}^{3}} \nabla_{y}^{s} f_{1} \cdot \nabla_{y}^{s} \Gamma d y
\end{aligned}
$$




$$
\begin{aligned}
& \frac{1}{2} \frac{d}{d \tau}\left\|\nabla_{y}^{s} \Lambda\right\|_{\mathbb{L}^{2}}^{2}+\left\|\nabla_{y}^{s+1} \Lambda\right\|_{\mathbb{L}^{2}}^{2}+\left(\frac{3}{4}-\frac{s}{2}+T^{*} e^{-\tau}+2 s \kappa\left(T^{*}\right)^{2 a+1} e^{-(2 a+1) \tau}\right)\left\|\nabla_{y}^{s} \Lambda\right\|_{\mathbb{L}^{2}}^{2} \\
& +\left(T^{*}\right)^{\frac{1}{2}} e^{-\frac{1}{2} \tau} \int_{\mathbb{R}^{3}}\left(\sum_{i=1}^{2} \nabla_{y}^{s}\left(h_{i} \partial_{y_{i}} d_{i}\right)-\nabla_{y}^{s} \Gamma\right) \cdot \nabla_{y}^{s} \Lambda d y \\
& =T^{*} e^{-\tau} \int_{\mathbb{R}^{3}} \nabla_{y}^{s} f_{2} \cdot \nabla_{y}^{s} \Lambda d y, \\
& \frac{1}{2} \frac{d}{d \tau}\left\|\nabla_{y}^{s} h_{1}\right\|_{\mathbb{L}^{2}}^{2}+v \sum_{i, j=1}^{3}\left\|\partial_{y_{i}} \nabla_{y}^{s} h_{j}\right\|_{\mathbb{L}^{2}}^{2}+\left(a+\frac{3}{4}-\frac{s}{2}\right)\left\|\nabla_{y}^{s} h_{1}\right\|_{\mathbb{L}^{2}}^{2} \\
& +k\left(T^{*}\right)^{2 a+1} e^{-(2 a+1) \tau} \int_{\mathbb{R}^{3}} \nabla_{y}^{s} h_{1} \cdot \nabla_{y}^{s} h_{2} d y \\
& +\left(T^{*}\right)^{\frac{1}{2}} e^{-\frac{1}{2} \tau} \sum_{i=1}^{3} \int_{\mathbb{R}^{3}} \nabla_{y}^{s} h_{1} \cdot\left(\nabla_{y}^{s} h_{i} \partial_{y_{i}} w_{1}+w_{i} \partial_{y_{i}} \nabla_{y}^{s} h_{1}\right) d y \\
& -\left(T^{*}\right)^{\frac{1}{2}} e^{-\frac{1}{2} \tau} \int_{\mathbb{R}^{3}} \nabla_{y}^{s} h_{1} \cdot \nabla_{y}^{s}\left(\Gamma \partial_{y_{1}} \Phi\right) d y \\
& =\int_{\mathbb{R}^{3}} \nabla_{y}^{s} h_{1} \cdot \tilde{g}_{1} d y, \\
& \frac{1}{2} \frac{d}{d \tau}\left\|\nabla_{y}^{s} h_{2}\right\|_{\mathbb{L}^{2}}^{2}+v \sum_{i, j=1}^{3}\left\|\partial_{y_{i}} \nabla_{y}^{s} h_{j}\right\|_{\mathbb{L}^{2}}^{2}+\left(a+\frac{3}{4}-\frac{s}{2}\right)\left\|\nabla_{y}^{s} h_{2}\right\|_{\mathbb{L}^{2}}^{2} \\
& -k\left(T^{*}\right)^{2 a+1} e^{-(2 a+1) \tau} \int_{\Omega} \nabla_{y}^{s} h_{1} \cdot \nabla_{y}^{s} h_{2} d y \\
& +\left(T^{*}\right)^{\frac{1}{2}} e^{-\frac{1}{2} \tau} \sum_{i=1}^{3} \int_{\Omega} \nabla_{y}^{s} h_{2} \cdot\left(\nabla_{y}^{s} h_{i} \partial_{y_{i}} w_{2}+w_{i} \partial_{y_{i}} \nabla_{y}^{s} h_{2}\right) d y \\
& -\left(T^{*}\right)^{\frac{1}{2}} e^{-\frac{1}{2} \tau} \int_{\Omega} \nabla_{y}^{s} h_{2} \cdot \nabla_{y}^{s}\left(\Gamma \partial_{y_{2}} \Phi\right) d y \\
& =\int_{\Omega} \nabla_{y}^{s} h_{2} \cdot \tilde{g}_{2} d y,
\end{aligned}
$$

and

$$
\begin{gathered}
\frac{1}{2} \frac{d}{d \tau}\left\|\nabla_{y}^{s} h_{3}\right\|_{\mathbb{L}^{2}}^{2}+v \sum_{i, j=1}^{3}\left\|\partial_{y_{i}} \nabla_{y}^{s} h_{j}\right\|_{\mathbb{L}^{2}}^{2}+\left(\frac{3}{4}-2 a-\frac{s}{2}\right)\left\|\nabla_{y}^{s} h_{3}\right\|_{\mathbb{L}^{2}}^{2} \\
+\left(T^{*}\right)^{\frac{1}{2}} e^{-\frac{1}{2} \tau} \sum_{i=1}^{3} \int_{\Omega} \nabla_{y}^{s} h_{3} \cdot\left(\nabla_{y}^{s} h_{i} \partial_{y_{i}} w_{3}+w_{i} \partial_{y_{i}} \nabla_{y}^{s} h_{3}\right) d y \\
-\left(T^{*}\right)^{\frac{1}{2}} e^{-\frac{1}{2} \tau} \int_{\Omega} \nabla_{y}^{s} h_{3} \cdot \nabla_{y}^{s}\left(\Gamma \partial_{y_{3}} \Phi\right) d y=\int_{\Omega} \nabla_{y}^{s} h_{3} \cdot \tilde{g}_{3} d y .
\end{gathered}
$$

Summing up (2.42)-(2.44), we have

$$
\begin{aligned}
& \frac{1}{2} \sum_{i=1}^{3} \frac{d}{d \tau}\left(\left\|\nabla_{y}^{s} \Gamma\right\|_{\mathbb{L}^{2}}^{2}+\left\|\nabla_{y}^{s} \Lambda\right\|_{\mathbb{L}^{2}}^{2}+\left\|\nabla_{y}^{s} h_{i}\right\|_{\mathbb{L}^{2}}^{2}\right)+\left\|\nabla_{y}^{s+1} \Gamma\right\|_{\mathbb{L}^{2}}^{2}+\left\|\nabla_{y}^{s+1} \Lambda\right\|_{\mathbb{L}^{2}}^{2} \\
& \quad+3 v \sum_{i, j=1}^{3}\left\|\partial_{y_{i}} \nabla_{y}^{s} h_{j}\right\|_{\mathbb{L}^{2}}^{2}+\left(\frac{3}{4}-\frac{s}{2}-T^{*} e^{-\tau} \sigma+2 s \kappa\left(T^{*}\right)^{2 a+1} e^{-(2 a+1) \tau}\right)\left\|\nabla_{y}^{s} \Gamma\right\|_{\mathbb{L}^{2}}^{2}
\end{aligned}
$$




$$
\begin{aligned}
& +2 \mu T^{*} e^{-\tau} \int_{\mathbb{R}^{3}} n\left|\nabla_{y} \Gamma\right|^{2} d y \\
& +\left(\frac{3}{4}-\frac{s}{2}+T^{*} e^{-\tau}+2 s \kappa\left(T^{*}\right)^{2 a+1} e^{-(2 a+1) \tau}\right)\left\|\nabla_{y}^{s} \Lambda\right\|_{\mathbb{L}^{2}}^{2} \\
& +\left(a+\frac{3}{4}-\frac{s}{2}\right)\left(\left\|\nabla_{y}^{s} h_{1}\right\|_{\mathbb{L}^{2}}^{2}+\left\|\nabla_{y}^{s} h_{2}\right\|_{\mathbb{L}^{2}}^{2}\right)+\left(\frac{3}{4}-2 a-\frac{s}{2}\right)\left\|\nabla_{y}^{s} h_{3}\right\|_{\mathbb{L}^{2}}^{2} \\
& +\left(T^{*}\right)^{\frac{1}{2}} e^{-\frac{1}{2} \tau} \int_{\mathbb{R}^{3}} \nabla_{y}^{s}\left(\mathbf{h} \cdot \nabla_{y} n\right) \cdot \nabla_{y}^{s} \Gamma d y+\chi \sum_{i=1}^{3} \int_{\mathbb{R}^{3}} \partial_{y_{i}} \nabla_{y}^{s}\left(\Gamma \partial_{y_{i}} c\right) \cdot \nabla_{y}^{s} \Gamma d y \\
& +\chi \int_{\mathbb{R}^{3}} \nabla_{y}^{s}\left(\nabla_{y} \cdot\left(n \nabla_{y} \Lambda\right)\right) \cdot \nabla_{y}^{s} \Gamma d y \\
& +\left(T^{*}\right)^{\frac{1}{2}} e^{-\frac{1}{2} \tau} \int_{\mathbb{R}^{3}}\left(\sum_{i=1}^{2} \nabla_{y}^{s}\left(h_{i} \partial_{y_{i}} d_{i}\right)-\nabla_{y}^{s} \Gamma\right) \cdot \nabla_{y}^{s} \Lambda d y \\
& +\left(T^{*}\right)^{\frac{1}{2}} e^{-\frac{1}{2} \tau} \sum_{i=1}^{3} \int_{\mathbb{R}^{3}}\left[\nabla_{y}^{s} h_{1} \cdot\left(\nabla_{y}^{s} h_{i} \partial_{y_{i}} w_{1}+w_{i} \partial_{y_{i}} \nabla_{y}^{s} h_{1}\right)\right. \\
& \left.+\nabla_{y}^{s} h_{2} \cdot\left(\nabla_{y}^{s} h_{i} \partial_{y_{i}} w_{2}+w_{i} \partial_{y_{i}} \nabla_{y}^{s} h_{2}\right)+\nabla_{y}^{s} h_{3} \cdot\left(\nabla_{y}^{s} h_{i} \partial_{y_{i}} w_{3}+w_{i} \partial_{y_{i}} \nabla_{y}^{s} h_{3}\right) d y\right] \\
& -\left(T^{*}\right)^{\frac{1}{2}} e^{-\frac{1}{2} \tau} \sum_{i=1}^{3} \int_{\mathbb{R}^{3}} \nabla_{y}^{s} h_{i} \cdot \nabla_{y}^{s}\left(\Gamma \partial_{y_{i}} \Phi\right) d y \\
& =T^{*} e^{-\tau} \int_{\mathbb{R}^{3}}\left(\nabla_{y}^{s} f_{1} \cdot \nabla_{y}^{s} \Gamma+\nabla_{y}^{s} f_{2} \cdot \nabla_{y}^{s} \Lambda\right) d y+\sum_{i=1}^{3} \int_{\mathbb{R}^{3}}\left(\nabla_{y}^{s} h_{i} \cdot \tilde{g}_{i}\right) d y .
\end{aligned}
$$

We now estimate each nonlinear term in (2.45). On the one hand, note that $(n, c, \mathbf{v})^{T} \in$ $\mathcal{B}_{R}$. We employ Young's inequality, $H^{\frac{5}{2}}\left(\mathbb{R}^{3}\right) \subset L^{\infty}\left(\mathbb{R}^{3}\right)$ and integrating by parts to derive

$$
\begin{aligned}
& \left.\left|2 \mu T^{*} e^{-\tau} \int_{\mathbb{R}^{3}} n\right| \nabla_{y} \Gamma\right|^{2} d y \mid \lesssim C_{R}\left\|\nabla_{y} \Gamma\right\|_{\mathbb{L}^{2}}^{2}, \\
& \left|\left(T^{*}\right)^{\frac{1}{2}} e^{-\frac{1}{2} \tau} \int_{\mathbb{R}^{3}} \nabla_{y}^{s}\left(\mathbf{h} \cdot \nabla_{y} n\right) \cdot \nabla_{y}^{s} \Gamma d y\right| \\
& \quad \lesssim C_{R}\left(\left\|\nabla_{y}^{s} \Gamma\right\|_{\mathbb{L}^{2}}^{2}+\sum_{i=1}^{3}\left(\left\|h_{i}\right\|_{\mathbb{L}^{2}}^{2}+\left\|\nabla_{y}^{s} h_{i}\right\|_{\mathbb{L}^{2}}^{2}\right)\right) \\
& \left|\chi \sum_{i=1}^{3} \int_{\mathbb{R}^{3}} \partial_{y_{i}} \nabla_{y}^{s}\left(\Gamma \partial_{y_{i}} c\right) \cdot \nabla_{y}^{s} \Gamma d y\right| \lesssim C_{R}\left(\left\|\nabla_{y}^{s+1} \Gamma\right\|_{\mathbb{L}^{2}}^{2}+\left\|\nabla_{y}^{s} \Gamma\right\|_{\mathbb{L}^{2}}^{2}\right), \\
& \left|\chi \int_{\mathbb{R}^{3}} \nabla_{y}^{s}\left(\nabla_{y} \cdot\left(n \nabla_{y} \Lambda\right)\right) \cdot \nabla_{y}^{s} \Gamma d y\right| \\
& \quad \lesssim C_{R}\left(\left\|\nabla_{y}^{s+1} \Gamma\right\|_{\mathbb{L}^{2}}^{2}+\left\|\nabla_{y}^{s+1} \Lambda\right\|_{\mathbb{L}^{2}}^{2}+\left\|\nabla_{y}^{s} \Gamma\right\|_{\mathbb{L}^{2}}^{2}+\left\|\nabla_{y}^{s} \Lambda\right\|_{\mathbb{L}^{2}}^{2}\right), \\
& \left|\left(T^{*}\right)^{\frac{1}{2}} e^{-\frac{1}{2} \tau} \int_{\mathbb{R}^{3}}\left(\sum_{i=1}^{2} \nabla_{y}^{s}\left(h_{i} \partial_{y_{i}} d_{i}\right)-\nabla_{y}^{s} \Gamma\right) \cdot \nabla_{y}^{s} \Lambda d y\right| \\
& \quad \lesssim \frac{\left(T^{*}\right)^{\frac{1}{2}}}{2} e^{-\frac{1}{2} \tau}\left(2\left\|\nabla_{y}^{s} \Lambda\right\|_{\mathbb{L}^{2}}^{2}+\left\|\nabla_{y}^{s} \Gamma\right\|_{\mathbb{L}^{2}}^{2}\right)+C_{R} \sum_{i=1}^{2}\left(\left\|h_{i}\right\|_{\mathbb{L}^{2}}^{2}+\left\|\nabla_{y}^{s} h_{i}\right\|_{\mathbb{L}^{2}}^{2}\right),
\end{aligned}
$$


and

$$
\begin{aligned}
& \left|\sum_{i=1}^{3} \int_{\mathbb{R}^{3}} \nabla_{y}^{s} h_{1} \cdot\left(\nabla_{y}^{s} h_{i} \partial_{y_{i}} w_{1}+w_{i} \partial_{y_{i}} \nabla_{y}^{s} h_{1}\right) d y\right| \\
& \lesssim\left(\sum_{k=1}^{3}\left(\left\|\partial_{y_{i}} w_{1}\right\|_{\mathbb{L}^{\infty}}+\left\|w_{i}\right\|_{\mathbb{L}^{\infty}}\right)\right) \sum_{i=1}^{3} \int_{\mathbb{R}^{3}}\left(\left|\nabla_{y}^{s} h_{i}\right|^{2}+\left|\partial_{y_{i}} \nabla_{y}^{s} h_{1}\right|^{2}\right) d y \\
& \lesssim C_{R} \sum_{i=1}^{3} \int_{\mathbb{R}^{3}}\left(\left|\nabla_{y}^{s} h_{i}\right|^{2}+\left|\partial_{y_{i}} \nabla_{y}^{s} h_{1}\right|^{2}\right) d y \\
& \left|\sum_{i=1}^{3} \int_{\mathbb{R}^{3}} \nabla_{y}^{s} h_{2} \cdot\left(\nabla_{y}^{s} h_{i} \partial_{y_{i}} w_{2}+w_{i} \partial_{y_{i}} \nabla_{y}^{s} h_{2}\right) d y\right| \\
& \lesssim\left(\sum_{i=1}^{3}\left(\left\|\partial_{y_{i}} w_{2}\right\|_{\mathbb{L}^{\infty}}+\left\|w_{i}\right\|_{\mathbb{L}^{\infty}}\right)\right) \sum_{i=1}^{3} \int_{\mathbb{R}^{3}}\left(\left|\nabla_{y}^{s} h_{i}\right|^{2}+\left|\partial_{y_{i}} \nabla_{y}^{s} h_{2}\right|^{2}\right) d y \\
& \lesssim C_{R} \sum_{k=1}^{3} \int_{\mathbb{R}^{3}}\left(\left|\nabla_{y}^{s} h_{i}\right|^{2}+\left|\partial_{y_{i}} \nabla_{y}^{s} h_{2}\right|^{2}\right) d y \\
& \left|\sum_{i=1}^{3} \int_{\mathbb{R}^{3}} \nabla_{y}^{s} h_{3} \cdot\left(\nabla_{y}^{s} h_{i} \partial_{y_{i}} w_{3}+w_{i} \partial_{y_{i}} \nabla_{y}^{s} h_{3}\right) d y\right| \\
& \lesssim\left(\sum_{i=1}^{3}\left(\left\|\partial_{y_{i}} w_{3}\right\|_{\mathbb{L}^{\infty}}+\left\|w_{i}\right\|_{\mathbb{L}^{\infty}}\right)\right) \\
& \times \sum_{i=1}^{3} \int_{\mathbb{R}^{3}}\left(\left|\nabla_{y}^{s} h_{i}\right|^{2}+\left|\partial_{y_{i}} \nabla_{y}^{s} h_{3}\right|^{2}\right) d y \\
& \lesssim C_{R} \sum_{i=1}^{3} \int_{\mathbb{R}^{3}}\left(\left|\nabla_{y}^{s} h_{i}\right|^{2}+\left|\partial_{y_{i}} \nabla_{y}^{s} h_{3}\right|^{2}\right) d y, \\
& \left|\left(T^{*}\right)^{\frac{1}{2}} e^{-\frac{1}{2} \tau} \sum_{i=1}^{3} \int_{\mathbb{R}^{3}} \nabla_{y}^{s} h_{i} \cdot \nabla_{y}^{s}\left(\Gamma \partial_{y_{i}} \Phi\right) d y\right| \\
& \lesssim C_{R}\left(\left\|\nabla_{y}^{s} \Gamma\right\|_{\mathbb{L}^{2}}^{2}+\sum_{i=1}^{3}\left\|\nabla_{y}^{s} h_{i}\right\|_{\mathbb{L}^{2}}^{2}\right)
\end{aligned}
$$

where the $C_{R}$ are a positive constants depending on $R$, which are small constants as $R$ is small.

On the other hand, by (2.18), we know the highest order derivatives on $h_{i}$ of $\partial_{y_{1}} \nabla_{y}^{s} \bar{f}$ is $s$. So we can use the standard Calderon-Zygmund theory, Young's inequality and integrating by parts to derive

$$
\left|\sum_{i=1}^{3} \int_{\mathbb{R}^{3}} \nabla_{y}^{s} h_{i} \cdot \partial_{y_{i}} \nabla_{y}^{s} \bar{f} d y\right| \lesssim C_{R}\left(\sum_{i=1}^{3}\left\|\nabla_{y}^{s} h_{i}\right\|_{\mathbb{L}^{2}}^{2}+\left\|\nabla_{y}^{s} \Gamma\right\|_{\mathbb{L}^{2}}^{2}\right),
$$


furthermore, by (2.37)-(2.39), we have

$$
\begin{aligned}
& \left|T^{*} e^{-\tau} \int_{\mathbb{R}^{3}}\left(\nabla_{y}^{s} f_{1} \cdot \nabla_{y}^{s} \Gamma+\nabla_{y}^{s} f_{2} \cdot \nabla_{y}^{s} \Lambda\right)\right| \\
& \quad \lesssim \frac{1}{2}\left(\sum_{i=1}^{2}\left\|\nabla_{y}^{s} f_{i}\right\|_{\mathbb{L}^{2}}^{2}+\left\|\nabla_{y}^{s} \Gamma\right\|_{\mathbb{L}^{2}}^{2}+\left\|\nabla_{y}^{s} \Lambda\right\|_{\mathbb{L}^{2}}^{2}\right), \\
& \left|\sum_{i=1}^{3} \int_{\mathbb{R}^{3}}\left(\nabla_{y}^{s} h_{i} \cdot \tilde{g}_{i}\right) d y\right| \\
& \quad \lesssim\left(C_{R, T^{*}}+\frac{1}{2}\right) \sum_{i=1}^{3}\left(\left\|\nabla_{y}^{s} h_{i}\right\|_{\mathbb{L}^{2}}^{2}+\left\|\nabla_{y}^{s} \Gamma\right\|_{\mathbb{L}^{2}}^{2}\right)+4 \sum_{i=1}^{3}\left\|\nabla_{y}^{s} g_{i}\right\|_{\mathbb{L}^{2}}^{2},
\end{aligned}
$$

where $C_{R, T^{*}}$ is a positive constant depending on $R, T^{*}$, which is a small constant as $R$ small.

Hence we can apply estimates (2.46)-(2.52) to (2.45), then

$$
\begin{aligned}
\frac{1}{2} \sum_{i=1}^{3} & \frac{d}{d \tau}\left(\left\|\nabla_{y}^{s} \Gamma\right\|_{\mathbb{L}^{2}}^{2}+\left\|\nabla_{y}^{s} \Lambda\right\|_{\mathbb{L}^{2}}^{2}+\left\|\nabla_{y}^{s} h_{i}\right\|_{\mathbb{L}^{2}}^{2}\right) \\
& +\left(1-C_{R}\right)\left\|\nabla_{y}^{s+1} \Gamma\right\|_{\mathbb{L}^{2}}^{2}+\left(1-C_{R}\right)\left\|\nabla_{y}^{s+1} \Lambda\right\|_{\mathbb{L}^{2}}^{2}+\left(3 v-C_{R}\right) \sum_{i, j=1}^{3}\left\|\partial_{y_{i}} \nabla_{y}^{s} h_{j}\right\|_{\mathbb{L}^{2}}^{2} \\
& +\left(\frac{3}{4}-\frac{s}{2}-T^{*} e^{-\tau}(b+\sigma)-\frac{\left(T^{*}\right)^{\frac{1}{2}}}{2} e^{-\frac{1}{2} \tau}-C_{R}\right)\|\Gamma\|_{\mathbb{L}^{2}}^{2} \\
& +\left(\frac{3}{4}-\frac{s}{2}+T^{*} e^{-\tau}(1-b)-\left(T^{*}\right)^{\frac{1}{2}} e^{-\frac{1}{2} \tau}-C_{R}\right)\|\Lambda\|_{\mathbb{L}^{2}}^{2} \\
& +\left(a+\frac{3}{4}-\frac{s}{2}-T^{*} e^{-\tau} b-C_{R}\right)\left(\left\|\nabla_{y}^{s} h_{1}\right\|_{\mathbb{L}^{2}}^{2}+\left\|\nabla_{y}^{s} h_{2}\right\|_{\mathbb{L}^{2}}^{2}\right) \\
& +\left(\frac{3}{4}-\frac{s}{2}-2 a-T^{*} e^{-\tau} b-C_{R}\right)\left\|\nabla_{y}^{s} h_{3}\right\|_{\mathbb{L}^{2}}^{2} \\
\lesssim & \frac{1}{2}\left(\left\|f_{1}\right\|_{\mathbb{L}^{2}}^{2}+\left\|f_{2}\right\|_{\mathbb{L}^{2}}^{2}\right)+4 \sum_{i=1}^{3}\left\|\nabla_{y}^{s} g_{i}\right\|_{\mathbb{L}^{2}}^{2} .
\end{aligned}
$$

Since $0<a \ll \frac{1}{8}$ and $0<s<\frac{3}{2}-5 a$ are constants, there exists a sufficient small positive constant $R$ such that

$$
\begin{aligned}
& 1-C_{R}>0, \quad 1-C_{R}>0, \quad 3 v-C_{R}>0, \\
& \frac{3}{4}-\frac{s}{2}-T^{*} e^{-\tau}(b+\sigma)-\frac{\left(T^{*}\right)^{\frac{1}{2}}}{2} e^{-\frac{1}{2} \tau}-C_{R}>0, \\
& \frac{3}{4}-\frac{s}{2}+T^{*} e^{-\tau}(1-b)-\left(T^{*}\right)^{\frac{1}{2}} e^{-\frac{1}{2} \tau}-C_{R}>0, \\
& a+\frac{3}{4}-\frac{s}{2}-T^{*} e^{-\tau} b-C_{R}>0, \\
& \frac{3}{4}-\frac{s}{2}-2 a-T^{*} e^{-\tau} b-C_{R}>0 .
\end{aligned}
$$


Hence, applying Gronwall's inequality to (2.53), there exists a positive constant $C_{R, T^{*}}$ depending on $R$ and $T^{*}$ such that

$$
\begin{aligned}
& \sum_{i=1}^{3}\left(\left\|\nabla_{y}^{s} \Gamma\right\|_{\mathbb{L}^{2}}^{2}+\left\|\nabla_{y}^{s} \Lambda\right\|_{\mathbb{L}^{2}}^{2}+\left\|\nabla_{y}^{s} h_{i}\right\|_{\mathbb{L}^{2}}^{2}\right) \\
& \lesssim e^{-C_{R, T^{*} \tau}} \sum_{i=1}^{3}\left(\left\|\nabla_{y}^{s} \Gamma_{0}\right\|_{\mathbb{L}^{2}}^{2}+\left\|\nabla_{y}^{s} \Lambda_{0}\right\|_{\mathbb{L}^{2}}^{2}+\left\|\nabla_{y}^{s} h_{0 i}\right\|_{\mathbb{L}^{2}}^{2}\right) \\
& \quad+e^{-C_{R, T^{*}} \tau} \int_{0}^{+\infty}\left(\left\|f_{1}\right\|_{\mathbb{L}^{2}}^{2}+\left\|f_{2}\right\|_{\mathbb{L}^{2}}^{2}+\sum_{i=1}^{3}\left\|\nabla_{y}^{s} g_{i}\right\|_{\mathbb{L}^{2}}^{2}\right) d \tau, \quad \forall \tau>0 .
\end{aligned}
$$

Furthermore, we have the following result.

Lemma 2.3 Let $0<a \ll \frac{1}{8}$ and $0<s<\frac{3}{2}-5$ a be constants. Assume that $\|\Phi\|_{\mathbb{H}^{s+5}\left(\mathbb{R}^{3}\right)} \lesssim R \ll$ $1, f_{i} \in \mathbb{C}^{1}\left((0,+\infty), \mathbb{H}^{s}\left(\mathbb{R}^{3}\right)\right)(i=1,2), \boldsymbol{g} \in \mathbb{C}^{1}\left((0,+\infty), H^{s}\left(\mathbb{R}^{3}\right)\right)$ and $(n, c, \boldsymbol{v})^{T} \in \mathcal{B}_{R}$. Then, for any $\tau>0$, the solution $(\Gamma, \Lambda, \boldsymbol{h})^{T}$ of the linearized coupled system (2.13)-(2.17) with the initial data (2.19) and condition (2.20) satisfies

$$
\begin{aligned}
& \int_{\mathbb{R}^{3}}\left(\left|\nabla_{y}^{s} \partial_{\tau} \Gamma\right|^{2}+\left|\nabla_{y}^{s} \partial_{\tau} \Lambda\right|^{2}+\sum_{i=1}^{3}\left|\nabla_{y}^{s} \partial_{\tau} h_{i}\right|^{2}\right) d y \\
& \lesssim e^{-C_{a, R, k, v, \mu, \delta} \tau} \int_{\mathbb{R}^{3}}\left(\left|\nabla_{y}^{s} \partial_{\tau} \Gamma_{0}\right|^{2}+\left|\nabla_{y}^{s} \partial_{\tau} \Lambda_{0}\right|^{2}+\sum_{i=1}^{3}\left|\nabla_{y}^{s} \partial_{\tau} h_{0 i}\right|^{2}\right) d y \\
& \quad+e^{-C_{a, R, k, v, \mu, \delta} \tau} \int_{0}^{+\infty}\left(\left\|\nabla_{y}^{s} \partial_{\tau} f_{1}\right\|_{\mathbb{L}^{2}}^{2}+\left\|\nabla_{y}^{s} \partial_{\tau} f_{2}\right\|_{\mathbb{L}^{2}}^{2}+\sum_{i=1}^{3}\left\|\nabla_{y}^{s} \partial_{\tau} g_{i}\right\|_{\mathbb{L}^{2}}^{2}\right) d \tau, \quad \forall \tau>0
\end{aligned}
$$

where $C_{a, R, \kappa, v, \mu, \delta}$ is a positive constant depending on the constants $a, R, \kappa, v, \mu, \delta$.

Proof Similar to getting the estimate in Lemma 2.2, we apply the operator $\partial_{\tau} \nabla_{y}^{s}$ to both sides of (2.8)-(2.10), then using a similar process to the proof of Lemma 2.2, we can obtain this result.

Proposition 2.1 Let $0<a \ll \frac{1}{8}$ and $0<s<\frac{3}{2}-5$ a be constants. Assume that $\|\Phi\|_{\mathbb{H}^{s+5}\left(\mathbb{R}^{3}\right)} \lesssim$ $R \ll 1, f_{i} \in \mathbb{C}^{1}\left((0,+\infty), \mathbb{H}^{s}\left(\mathbb{R}^{3}\right)\right)(i=1,2), \boldsymbol{g} \in \mathbb{C}^{1}\left((0,+\infty), H^{s}\left(\mathbb{R}^{3}\right)\right)$ and $(n, c, \boldsymbol{v})^{T} \in \mathcal{B}_{R}$. Then, for any $\tau>0$, the linearized coupled system (2.13)-(2.17) with the initial data (2.19) and condition (2.20) admits a solution

$$
\begin{aligned}
& \Gamma \in \mathcal{C}_{0}^{s}:=\bigcap_{i=0}^{1} \mathbb{C}^{i}\left((0,+\infty) ; \mathbb{H}^{s-i}\right), \\
& \Lambda \in \mathcal{C}_{0}^{s}:=\bigcap_{i=0}^{1} \mathbb{C}^{i}\left((0,+\infty) ; \mathbb{H}^{s-i}\right), \\
& \boldsymbol{h} \in \overline{\mathcal{C}}_{0}^{s}:=\bigcap_{i=0}^{1} \mathbb{C}^{i}\left((0,+\infty) ; H^{s-i}\right),
\end{aligned}
$$


Moreover,

$$
\begin{aligned}
& \|\Gamma\|_{\mathcal{C}_{0}^{s}}^{2}+\|\Lambda\|_{\mathcal{C}_{0}^{s}}^{2}+\|\boldsymbol{h}\|_{\overline{\mathcal{C}}_{0}^{s}}^{2} \lesssim\left\|\Gamma_{0}\right\|_{\mathcal{C}_{0}^{s}}^{2}+\left\|\Lambda_{0}\right\|_{\mathcal{C}_{0}}^{2}+\left\|\boldsymbol{h}_{0}\right\|_{\overline{\mathcal{C}}_{0}^{s}}^{2}+\left\|f_{1}\right\|_{\mathcal{C}_{0}^{s}}^{2}+\left\|f_{2}\right\|_{\mathcal{C}_{0}^{s}}^{2}+\|\boldsymbol{g}\|_{\overline{\mathcal{C}}_{0}^{s}}^{2}, \\
& \quad \forall \tau>0 .
\end{aligned}
$$

Proof Let $\mathbb{P}$ be the Leray projector onto the space of divergence free functions. We apply the Leray projector to system (2.5), we have

$$
\left\{\begin{array}{l}
\Gamma_{t}-\Delta \Gamma+(2 \mu n-\sigma) \Gamma+(\mathbf{v}+\overline{\mathbf{u}}) \cdot \nabla \Gamma+\mathbf{h} \cdot \nabla n+\chi \nabla \cdot[\Gamma \nabla c+n \nabla \Lambda]=f_{1}(t, x) \\
\Lambda_{t}-\Delta \Lambda+\Lambda+(\mathbf{v}+\overline{\mathbf{u}}) \cdot \nabla \Lambda+\mathbf{h} \cdot \nabla c-\Gamma=f_{2}(t, x), \\
\mathbf{h}_{t}-v \mathbb{P} \Delta \mathbf{h}+\mathbb{P}(\mathbf{h} \cdot \nabla(\overline{\mathbf{u}}+\mathbf{v})+(\overline{\mathbf{u}}+\mathbf{v}) \cdot \nabla \mathbf{h}-\Gamma \nabla \Phi)=\mathbb{P} \mathbf{g}(t, x) .
\end{array}\right.
$$

In the similarity coordinates (2.12), we can rewrite the linear system (2.55) as

$$
\partial_{\tau} U+(\mathcal{A}+\mathcal{N}) U=T^{*} e^{-\tau} F
$$

where $U:=\left(\Gamma, \Lambda, h_{1}, h_{2}, h_{3}\right)^{T}, \mathcal{N}(U):=\left(\mathbb{M}_{1}, \mathbb{M}_{2}, \mathbb{N}_{1}, \mathbb{N}_{2}, \mathbb{N}_{3}\right)^{T}, F:=\left(f_{1}, f_{2}, \mathbb{P} g_{1}, \mathbb{P} g_{2}, \mathbb{P} g_{3}\right)^{T}$ and the matrix operator is given by

$$
\mathcal{A}:=\left(\begin{array}{ccccc}
-\mu \triangle_{y} & 0 & 0 & 0 & 0 \\
0 & -\delta \triangle_{y} & 0 & 0 & 0 \\
0 & 0 & -v \mathbb{P} \triangle_{y} & 0 & 0 \\
0 & 0 & 0 & -v \mathbb{P} \triangle_{y} & 0 \\
0 & 0 & 0 & 0 & -v \mathbb{P} \triangle_{y}
\end{array}\right)_{5 \times 5},
$$

and

$$
\begin{aligned}
\mathbb{M}_{1}(\Gamma, \Lambda, \mathbf{h}):= & -\triangle_{y} \Gamma-\frac{y}{2} \cdot \nabla_{y} \Gamma+T^{*} e^{-\tau}(2 \mu n-\sigma) \Gamma+a y_{1} \partial_{y_{1}} \Gamma+a y_{2} \partial_{y_{2}} \Gamma \\
& -2 a y_{3} \partial_{y_{3}} \Gamma+k\left(T^{*}\right)^{2 a+1} e^{-(2 a+1) \tau}\left(y_{2} \partial_{y_{1}} \Gamma+y_{1} \partial_{y_{2}} \Gamma\right) \\
& +\left(T^{*}\right)^{\frac{1}{2}} e^{-\frac{1}{2} \tau} \mathbf{h} \cdot \nabla_{y} n+\chi \sum_{i=1}^{3} \partial_{y_{i}}\left(\Gamma \partial_{y_{i}} c\right)+\chi \nabla_{y} \cdot\left(n \nabla_{y} \Lambda\right), \\
\mathbb{M}_{2}(\Gamma, \Lambda, \mathbf{h}):= & -\frac{y}{2} \cdot \nabla_{y} \Lambda+T^{*} e^{-\tau} \Lambda+a y_{1} \partial_{y_{1}} \Lambda+a y_{2} \partial_{y_{2}} \Lambda-2 a y_{3} \partial_{y_{3}} \Lambda \\
& +k\left(T^{*}\right)^{2 a+1} e^{-(2 a+1) \tau}\left(y_{2} \partial_{y_{1}} \Lambda+y_{1} \partial_{y_{2}} \Lambda\right)+\left(T^{*}\right)^{\frac{1}{2}} e^{-\frac{1}{2} \tau}\left(\sum_{i=1}^{2} h_{i} \partial_{y_{i}} c_{i}-\Gamma\right), \\
\mathbb{N}_{1}(\Gamma, \Lambda, \mathbf{h}):= & -\frac{y}{2} \cdot \nabla_{y} h_{1}+a h_{1}+a y_{1} \partial_{y_{1}} h_{1}+a y_{2} \partial_{y_{2}} h_{1}-2 a y_{3} \partial_{y_{3}} h_{1} \\
& +k\left(T^{*}\right)^{2 a+1} e^{-(2 a+1) \tau}\left(h_{2}+y_{2} \partial_{y_{1}} h_{1}+y_{1} \partial_{y_{2}} h_{1}\right)-\left(T^{*}\right)^{\frac{1}{2}} e^{-\frac{1}{2} \tau} \Gamma \partial_{y_{1}} \Phi \\
& +\left(T^{*}\right)^{\frac{1}{2}} e^{-\frac{1}{2} \tau} \sum_{i=1}^{3}\left(h_{i} \partial_{y_{i}} w_{1}+w_{i} \partial_{y_{i}} h_{1}\right),
\end{aligned}
$$




$$
\begin{aligned}
\mathbb{N}_{2}(\Gamma, \Lambda, \mathbf{h}):= & -\frac{y}{2} \cdot \nabla_{y} h_{2}+a h_{2}+a y_{1} \partial_{y_{1}} h_{2}+a y_{2} \partial_{y_{2}} h_{2}-2 a y_{3} \partial_{y_{3}} h_{2} \\
& +k\left(T^{*}\right)^{2 a+1} e^{-(2 a+1) \tau}\left(-h_{1}+y_{2} \partial_{y_{1}} h_{2}-y_{1} \partial_{y_{2}} h_{2}\right)-\left(T^{*}\right)^{\frac{1}{2}} e^{-\frac{1}{2} \tau} \Gamma \partial_{y_{2}} \Phi \\
& +\left(T^{*}\right)^{\frac{1}{2}} e^{-\frac{1}{2} \tau} \sum_{i=1}^{3}\left(h_{i} \partial_{y_{i}} w_{2}+w_{i} \partial_{y_{i}} h_{2}\right), \\
\mathbb{N}_{3}(\Gamma, \Lambda, \mathbf{h}):= & -\frac{y}{2} \cdot \nabla_{y} h_{3}-a h_{3}+a y_{1} \partial_{y_{1}} h_{3}+a y_{2} \partial_{y_{2}} h_{3}-2 a y_{3} \partial_{y_{3}} h_{3} \\
& +k\left(T^{*}\right)^{2 a+1} e^{-(2 a+1) \tau}\left(y_{2} \partial_{y_{1}} h_{3}-y_{1} \partial_{y_{2}} h_{3}\right)-\left(T^{*}\right)^{\frac{1}{2}} e^{-\frac{1}{2} \tau} \Gamma \partial_{y_{3}} \Phi \\
& +\left(T^{*}\right)^{\frac{1}{2}} e^{-\frac{1}{2} \tau} \sum_{i=1}^{3}\left(h_{i} \partial_{y_{i}} w_{3}+w_{i} \partial_{y_{i}} h_{3}\right) .
\end{aligned}
$$

Obviously, there is no singular coefficient in the linear operator $\mathcal{A}+\mathcal{N}$ by noticing (2.55). We follow the idea of [38] to show the linear operator $\mathcal{A}+\mathcal{N}$ generate a strongly continuous semigroup $e^{(\mathcal{A}+\mathcal{N}) \tau}$ in Sobolev space $\mathbb{H}^{s}\left(\mathbb{R}^{3}\right) \times \mathbb{H}^{s}\left(\mathbb{R}^{3}\right) \times H^{s}\left(\mathbb{R}^{3}\right)$. To see this, by the same process as getting (2.53), for the constants $0<a \ll \frac{1}{8}$ and $0<s<\frac{3}{2}-5 a$, we can obtain

$$
\begin{aligned}
\int_{\Omega} \nabla_{y}^{s} U \cdot \nabla_{y}^{s}((\mathcal{A}+\mathcal{N}) U) d y \\
\lesssim-\left(\frac{3}{4}-\frac{s}{2}-T^{*} e^{-\tau}(b+\sigma)-\frac{\left(T^{*}\right)^{\frac{1}{2}}}{2} e^{-\frac{1}{2} \tau}-C_{R}\right)\|\Gamma\|_{\mathbb{L}^{2}}^{2} \\
-\left(\frac{3}{4}-\frac{s}{2}+T^{*} e^{-\tau}(1-b)-\left(T^{*}\right)^{\frac{1}{2}} e^{-\frac{1}{2} \tau}-C_{R}\right)\|\Lambda\|_{\mathbb{L}^{2}}^{2} \\
-\left(a+\frac{3}{4}-\frac{s}{2}-T^{*} e^{-\tau} b-C_{R}\right)\left(\left\|\nabla_{y}^{s} h_{1}\right\|_{\mathbb{L}^{2}}^{2}+\left\|\nabla_{y}^{s} h_{2}\right\|_{\mathbb{L}^{2}}^{2}\right) \\
-\left(\frac{3}{4}-\frac{s}{2}-2 a-T^{*} e^{-\tau} b-C_{R}\right)\left\|\nabla_{y}^{s} h_{3}\right\|_{\mathbb{L}^{2}}^{2}
\end{aligned}
$$

and

$$
\begin{aligned}
& \frac{3}{4}-\frac{s}{2}-T^{*} e^{-\tau}(b+\sigma)-\frac{\left(T^{*}\right)^{\frac{1}{2}}}{2} e^{-\frac{1}{2} \tau}-C_{R}>0, \\
& \frac{3}{4}-\frac{s}{2}+T^{*} e^{-\tau}(1-b)-\left(T^{*}\right)^{\frac{1}{2}} e^{-\frac{1}{2} \tau}-C_{R}>0, \\
& a+\frac{3}{4}-\frac{s}{2}-T^{*} e^{-\tau} b-C_{R}>0, \\
& \frac{3}{4}-\frac{s}{2}-2 a-T^{*} e^{-\tau} b-C_{R}>0 .
\end{aligned}
$$

Hence by (2.56), we get

$$
\int_{\Omega} \nabla_{y}^{s} U \cdot \nabla_{y}^{s}((\mathcal{A}+\mathcal{N}) U) d y \leq 0
$$

Hence the linear operator $\mathcal{A}+\mathcal{N}$ is a linear dissipative operator in $\mathbb{H}^{s}\left(\mathbb{R}^{3}\right) \times \mathbb{H}^{s}\left(\mathbb{R}^{3}\right) \times$ $H^{s}\left(\mathbb{R}^{3}\right)$. Moreover, if we set

$$
(\mathcal{A}+\mathcal{N}) U=0
$$


then, by (2.56), we know the linear operator $\mathcal{A}+\mathcal{N}$ is injective. Furthermore, we can verify that this linear operator is surjective by using the standard theory of elliptic-type equations of the general order. Thus the linear operator $\mathcal{A}+\mathcal{N}$ can generate a strongly continuous semigroup $e^{(\mathcal{A}+\mathcal{N}) \tau}$ in Sobolev space $\mathbb{H}^{s}\left(\mathbb{R}^{3}\right) \times \mathbb{H}^{s}\left(\mathbb{R}^{3}\right) \times H^{s}\left(\mathbb{R}^{3}\right)$ by the Lumer-Phillips theorem [23]. Therefore, the linear system (2.55) admits a solution

$$
\begin{aligned}
& \Gamma \in \mathcal{C}_{0}^{s}:=\bigcap_{i=0}^{1} \mathbb{C}^{i}\left((0,+\infty) ; \mathbb{H}^{s-i}\right), \\
& \Lambda \in \mathcal{C}_{0}^{s}:=\bigcap_{i=0}^{1} \mathbb{C}^{i}\left((0,+\infty) ; \mathbb{H}^{s-i}\right), \\
& \mathbf{h} \in \overline{\mathcal{C}}_{0}^{s}:=\bigcap_{i=0}^{1} \mathbb{C}^{i}\left((0,+\infty) ; H^{s-i}\right),
\end{aligned}
$$

Furthermore, it follows from Lemmas 2.2-2.3 that (2.54) holds.

Recalling the self-similarity coordinates (2.12), the original coordinate can be expressed by the self-similarity coordinates as follows:

$$
t=T\left(1-e^{-\tau}\right), \quad x=y \sqrt{T^{*}-t},
$$

so we can directly use Proposition 2.1 to get the following result.

Proposition 2.2 Let $0<a \ll \frac{1}{8}$ and $0<s<\frac{3}{2}-5$ a be constants. Assume that $\|\Phi\|_{\mathbb{H}^{\mathrm{S}+5}\left(\mathbb{R}^{3}\right)} \lesssim$ $R \ll 1, f_{i} \in \mathbb{C}^{1}\left(\left(0, T^{*}\right), \mathbb{H}^{s}\left(\mathbb{R}^{3}\right)\right)(i=1,2), \boldsymbol{g} \in \mathbb{C}^{1}\left(\left(0, T^{*}\right), H^{s}\left(\mathbb{R}^{3}\right)\right)$ and $(n, c, \boldsymbol{v})^{T} \in \mathcal{B}_{R}$. Then the linearized coupled system (2.5) with the initial data (2.2) and condition (2.3) admits a local solution

$$
\begin{aligned}
& \Gamma \in \mathcal{C}_{0}^{s}:=\bigcap_{i=0}^{1} \mathbb{C}^{i}\left(\left(0, T^{*}\right) ; \mathbb{H}^{s-i}\left(\mathbb{R}^{3}\right)\right), \\
& \Lambda \in \mathcal{C}_{0}^{s}:=\bigcap_{i=0}^{1} \mathbb{C}^{i}\left(\left(0, T^{*}\right) ; \mathbb{H}^{s-i}\left(\mathbb{R}^{3}\right)\right), \\
& \boldsymbol{h} \in \overline{\mathcal{C}}_{0}^{s}:=\bigcap_{i=0}^{1} \mathbb{C}^{i}\left(\left(0, T^{*}\right) ; H^{s-i}\left(\mathbb{R}^{3}\right)\right),
\end{aligned}
$$

Moreover,

$$
\begin{aligned}
& \|\Gamma\|_{\mathcal{C}_{0}^{s}}^{2}+\|\Lambda\|_{\mathcal{C}_{0}^{s}}^{2}+\|\boldsymbol{h}\|_{\mathcal{C}_{0}^{s}}^{2} \lesssim\left\|\Gamma_{0}\right\|_{\mathcal{C}_{0}^{s}}^{2}+\left\|\Lambda_{0}\right\|_{\mathcal{C}_{0}}^{2}+\left\|\boldsymbol{h}_{0}\right\|_{\mathcal{C}_{0}^{s}}^{2}+\left\|f_{1}\right\|_{\mathcal{C}_{0}^{s}}^{2}+\left\|f_{2}\right\|_{\mathcal{C}_{0}^{s}}^{2}+\|\boldsymbol{g}\|_{\mathcal{C}_{0}^{s}}^{2}, \\
& \quad \forall t \in\left(0, T^{*}\right) .
\end{aligned}
$$

\section{Acknowledgements}

We are grateful to the anonymous referees who read the paper very carefully and made numerous suggestions for improving the presentation. 
Availability of data and materials

Data sharing is not applicable to this article as no datasets were generated or analyzed during the current study.

\section{Competing interests}

The authors declare that they have no competing interests.

\section{Authors' contributions}

The authors contributed equally and significantly in writing this paper. All authors read and approved the final manuscript.

\section{Publisher's Note}

Springer Nature remains neutral with regard to jurisdictional claims in published maps and institutional affiliations.

Received: 4 February 2021 Accepted: 8 April 2021 Published online: 16 April 2021

\section{References}

1. Bedrossian, J., Germain, P., Masmoudi, N.: On the stability threshold for the 3D Couette flow in Sobolev regularity. Ann. Math. 185, 541-608 (2017)

2. Bellomo, N., Bellouquid, A., Chouhad, N.: From a multiscale derivation of nonlinear cross-diffusion models to Keller-Segel models in a Navier-Stokes fluid. Math. Models Methods Appl. Sci. 26, 2041-2069 (2016)

3. Bellomo, N., Bellouquid, A., Tao, Y., Winkler, M.: Toward a mathematical theory of Keller-Segel models of pattern formation in biological tissues. Math. Models Methods Appl. Sci. 25, 1663-1763 (2015)

4. Caffarelli, L., Kohn, R.V., Nirenberg, L.: Partial regularity of suitable weak solutions of the Navier-Stokes equations. Commun. Pure Appl. Math. 35, 771-831 (1982)

5. Cannone, M., Planchon, F.: Self-similar solutions for Navier-Stokes equations in $\mathbb{R}^{3}$. Commun. Partial Differ. Equ. 21, 179-193 (1996)

6. Chae, M., Kang, K., Lee, J.: Global existence and temporal decay in Keller-Segel models coupled to fluid equations. Commun. Partial Differ. Equ. 39, 1205-1235 (2014)

7. Duan, R.J., Lorz, A., Markowich, P.A.: Global solutions to the coupled chemotaxis-fluid equations. Commun. Partial Differ. Equ. 35, 1635-1673 (2010)

8. Farwig, R., Kanamaru, R.: Optimality of Serrin type extension criteria to the Navier-Stokes equations. Adv. Nonlinear Anal. 10, 1071-1085 (2021)

9. Fefferman, C.L.: Existence and smoothness of the Navier-Stokes equations. Millenn. Prize Probl. 57-67 (2006)

10. Fu, Y.Q., Rădulescu, V.D., Zhang, B.L.: Hodge decomposition of variable exponent spaces of Clifford-valued functions and applications to Dirac and Stokes equations. Commun. Math. Appl. 70, 691-704 (2015)

11. Hillen, T., Painter, K.J.: A user's guide to PDE models for chemotaxis. J. Math. Biol. 58, 183-217 (2009)

12. Kiselev, A., Ryzhik, L.: Biomixing by chemotaxis and enhancement of biological reactions. Commun. Partial Differ. Equ. 37, 298-318 (2012)

13. Kiselev, A., Ryzhik, L.: Biomixing by chemotaxis and efficiency of biological reactions: the critical reaction case. J. Math. Phys. 53, 115609 (2012)

14. Kiselev, A., Xu, X.: Suppression of chemotactic explosion by mixing. Arch. Ration. Mech. Anal. 222, 1077-1112 (2016)

15. Lankeit, J.: Eventual smoothness and asymptotics in a three-dimensional chemotaxis system with logistic source. J. Differ. Equ. 258, 1158-1191 (2015)

16. Leray, J.: Sur le mouvement d'un liquide visqueux emplissant l'espace. Acta Math. 63, 193-248 (1934)

17. Li, H.Y., Zhao, X., Yan, W.P.: Bifurcation of time periodic solutions for the incompressible flow of nematic liquid crystals in three dimension. Adv. Nonlinear Anal. 9, 1315-1332 (2020)

18. Liu, J.G., Lorz, A.: A coupled chemotaxis-fluid model: global existence. Ann. Inst. Henri Poincaré, Anal. Non Linéaire 28 643-652 (2011)

19. Lorz, A.: Coupled chemotaxis fluid model. Math. Models Methods Appl. Sci. 20, 987-1004 (2010)

20. Mittal, N., Budrene, E.O., Brenner, M.E., van Oudenaarden, A.: Motility of Escherichia coli cells in clusters formed by chemotactic aggregation. Proc. Natl. Acad. Sci. USA 100, 13229-13263 (2003)

21. Mohammed, A., Radulescu, V., Vitolo, A.: Blow up solutions for full nonlinear equations: existence, asymptotic estimates and uniqueness. Adv. Nonlinear Anal. 9, 39-64 (2020)

22. Necas, J., Ruzicka, M., Sverák, V.: On Leray's self-similar solutions of the Navier-Stokes equations. Acta Math. 176 283-294 (1996)

23. Pazy, A.: Semigroups of Linear Operators and Applications to Partial Differential Equations. Springer, New York (1983)

24. Tang, X.H., Chen, S.T.: Singularly perturbed Choquard equations with nonlinearity satisfying Berestycki-Lions assumptions. Adv. Nonlinear Anal. 9, 413-437 (2020)

25. Tao, Y., Winkler, M.: Locally bounded global solutions in a three-dimensional chemotaxis-Stokes system with nonlinear diffusion. Ann. Inst. Henri Poincaré, Anal. Non Linéaire 30, 157-178 (2013)

26. Tuval, I., Cisneros, L., Dombrowski, C., Wolgemuth, C.W., Kessler, J.O., Goldstein, R.E.: Bacterial swimming and oxygen transport near contact lines. Proc. Natl. Acad. Sci. USA 102, 2277-2282 (2005)

27. Wang, Y.L.: Global weak solutions in a three dimensional Keller-Segel-Navier-Stokes system with subcritical sensitivity. Math. Models Methods Appl. Sci. 27, 2745-2780 (2017)

28. Wang, Y.L., Winkler, M., Xiang, Z.Y.: The small-convection limit in a two-dimensional chemotaxis-Navier-Stokes system. Math. Z. 289, 71-108 (2018)

29. Winkler, M.: Global large-data solutions in a chemotaxis-(Navier-)Stokes system modeling cellular swimming in fluid drops. Commun. Partial Differ. Equ. 37, 319-351 (2012)

30. Winkler, M.: Finite-time blow-up in the higher-dimensional parabolic-parabolic Keller-Segel system. J. Math. Pures Appl. 100, 748-767 (2013) 
31. Winkler, M.: Stabilization in a two dimensional chemotaxis-Navier-Stokes system. Arch. Ration. Mech. Anal. 2011, 455-487 (2014)

32. Winkler, M.: How far can chemotactic cross-diffusion enforce exceeding carrying capacities? J. Nonlinear Sci. 24 809-855 (2014)

33. Winkler, M.: Global weak solutions in a three-dimensional chemotaxis-Navier-Stokes system. Ann. Inst. Henri Poincaré, Anal. Non Linéaire 33, 1329-1352 (2016)

34. Winkler, M.: How far do chemotaxis-driven forces influence regularity in the Navier-Stokes system? Trans. Am. Math. Soc. 369, 3067-3125 (2017)

35. Winkler, M.: A three dimensional Keller-Segel-Navier-Stokes system with logistic source: global weak solutions and asymptotic stabilization. J. Funct. Anal. 276, 1339-1401 (2019)

36. Yan, W.P.: Two family of explicit blowup solutions for 3 D incompressible Navier-Stokes equations (2018) arXiv:1807.05425

37. Yan, W.P.: Nonlinear stability of infinite energy blowup solutions for the 3D incompressible Navier-Stokes equations in $\mathbb{R}^{n}$. Preprint

38. Yudovich, V.I.: The Linearization Method in Hydrodynamical Stability Theory. Translations of Mathematical Monographs, vol. 74. Am. Math. Soc., Providence (1989)

39. Zhang, B.L., Fu, Y.Q., Rădulescu, V.D.: The stationary Navier-Stokes equations in variable exponent spaces of Clifford-valued functions. Adv. Appl. Clifford Algebras 24, 231-252 (2014)

40. Zhang, Q., Zheng, X.: Global well-posedness for the two dimensional incompressible Chemotaxis-Navier-Stokes equations. SIAM J. Math. Anal. 46, 3078-3105 (2014)

\section{Submit your manuscript to a SpringerOpen ${ }^{\circ}$} journal and benefit from:

- Convenient online submission

- Rigorous peer review

- Open access: articles freely available online

- High visibility within the field

- Retaining the copyright to your article

Submit your next manuscript at $\boldsymbol{\nabla}$ springeropen.com 